\title{
Transient Pressure Behavior of Reservoirs with Discrete Conductive Faults and Fractures
}

\author{
Denis Biryukov • Fikri J. Kuchuk
}

Received: 27 November 2011 / Accepted: 25 June 2012 / Published online: 19 July 2012

(C) The Author(s) 2012. This article is published with open access at Springerlink.com

\begin{abstract}
Fractures and faults are common features of many well-known reservoirs. They create traps, serve as conduits to oil and gas migration, and can behave as barriers or baffles to fluid flow. Naturally fractured reservoirs consist of fractures in igneous, metamorphic, sedimentary rocks (matrix), and formations. In most sedimentary formations both fractures and matrix contribute to flow and storage, but in igneous and metamorphic rocks only fractures contribute to flow and storage, and the matrix has almost zero permeability and porosity. In this study, we present a mesh-free semianalytical solution for pressure transient behavior in a $2 \mathrm{D}$ infinite reservoir containing a network of discrete and/or connected finite- and infinite-conductivity fractures. The proposed solution methodology is based on an analytical-element method and thus can be easily extended to incorporate other reservoir features such as sealing or leaky faults, domains with altered petrophysical properties (for example, fluid permeability or reservoir porosity), and complicated reservoir boundaries. It is shown that the pressure behavior of discretely fractured reservoirs is considerably different from the well-known Warren and Root dual-porosity reservoir model behavior. The pressure behavior of discretely fractured reservoirs shows many different flow regimes depending on fracture distribution, its intensity and conductivity. In some cases, they also exhibit a dual-porosity reservoir model behavior.
\end{abstract}

Keywords Discretely fractured reservoirs · Discretely faulted reservoirs · Pressure diffusion in fractured porous media - Uniform-pressure boundary condition . Pressure transient well testing 


\section{List of symbols}

\section{Variables}

$\begin{array}{ll}c_{\mathrm{t}} & \text { Compressibility } \\ C_{n}^{(k)} & \text { Gegenbauer polynomials } \\ \mathrm{E}_{1}, \mathrm{E}_{2} & \text { Exponential integrals } \\ \mathrm{erf} & \text { Error function } \\ F & \text { Fracture conductivity } \\ h & \text { Formation thickness } \\ \mathrm{H} & \text { Heaviside step function } \\ \mathrm{K} & \text { Modified Bessel Function of the second kind } \\ k & \text { Permeability } \\ l & \text { Fracture/fault half-length } \\ p & \text { Pressure } \\ q & \text { Flow rate or flux density } \\ r & \text { Radius or radial coordinate } \\ s & \text { Laplace transform variable } \\ T & \text { Chebyshev polynomial } \\ t & \text { Time } \\ x & \text { Coordinate } \\ y & \text { Coordinate } \\ z & \text { Vertical coordinate } \\ \alpha & \text { Transmissibility } \\ \delta & \text { The Dirac delta functional } \\ \eta & \text { Diffusivity for pressure } \\ \mu & \text { Viscosity } \\ \phi & \text { Porosity } \\ \tau & \text { Dummy variable } \\ \xi & \text { Dummy variable } \\ & \\ & \end{array}$

\section{Subscripts}

D Dimensionless

f Fracture

o Initial or original

w Wellbore

\section{Superscripts}

- Laplace transform

\section{Introduction}

Naturally fractured or fissured reservoirs can be described as sedimentary formations fractured by tectonic, thermal, and/or chemical processes. They consist of fractures, and igneous, metamorphic, or sedimentary rocks (matrix). In most sedimentary formations both fractures and matrix contribute to flow and storage, but in igneous and metamorphic rocks only fractures contribute to flow and storage, and the matrix has almost zero permeability and porosity. 
(a)

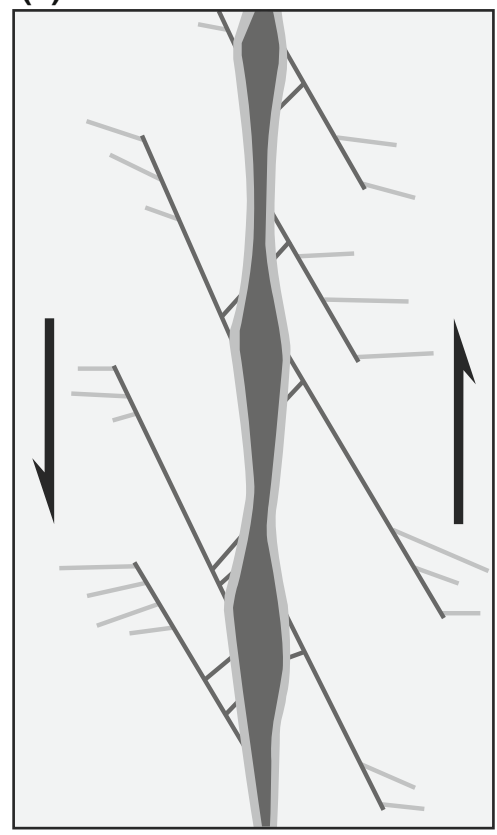

(b)

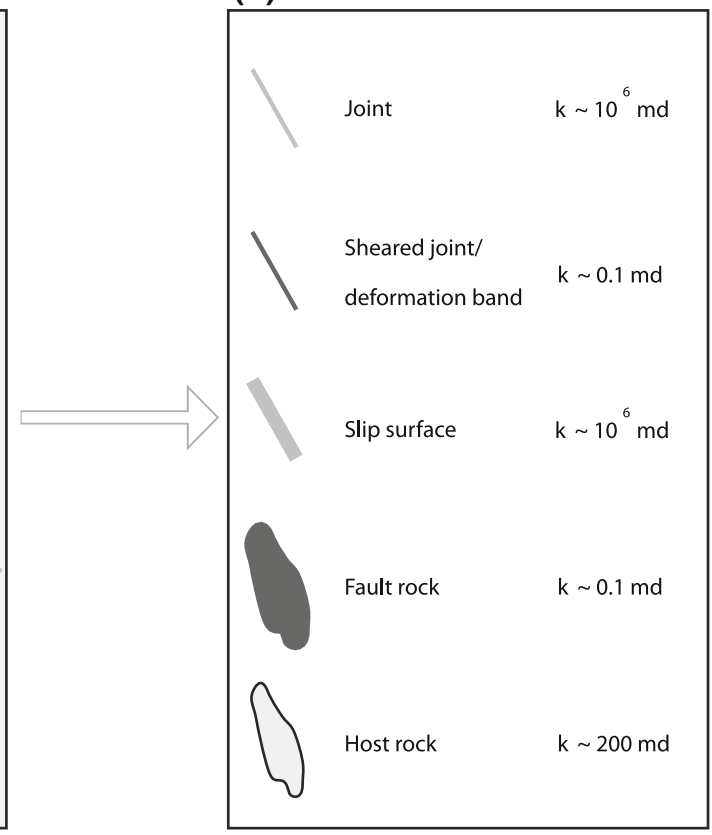

Fig. 1 Fault permeabilities: a field characterization of the fault element geometry and b laboratory analysis for the fault element permeability, after Jourde et al. (2002) reprinted by permission of AAPG

Faulted reservoirs are also created by the similar geological processes. Faults and fractures create traps, and serve as conduits to gas, oil, and water migration, and can behave as barriers or baffles to fluid flow.

Faults and fractures occur frequently in reservoirs and profoundly affect the fluid flow. They can either impede or enhance fluid flow dramatically, thereby playing an important role in migration, entrapment, production of gas and oil, and in all secondary and tertiary recovery processes. Complexities arise even further when faults provide conduits for injected and/or aquifer water that may further enhance or reduce their conductivities. Although in the well testing literature faults are usually considered to be sealing or leaky, in general, they can be highly conductive like natural fractures (Aydin 2000; Flodin et al. 2001; Jourde et al. 2002). Fault conductivities or permeabilities can be highly variable and significantly lower or higher than those of the host rock (Aydin 2000; Flodin et al. 2001; Jourde et al. 2002), as shown by Figs. 1 and 2. As can be observed in these figures, fault permeabilities can be a few orders of magnitude higher than the host rock permeabilities: from zero to almost an open-crack infinite permeability. As stated in these papers, fault permeabilities can be highly anisotropic. Therefore, like natural fractures, faults can create a continuous or discrete conductive network in the reservoirs, where the pressure transient behavior of the system can be similar to that of the naturally fractured reservoirs. It should be also stated that faults can create highly compartmentalized reservoirs, where they become barriers to flow.

In this article, the term Naturally fractured reservoirs should be applied to both faulted and fractured reservoirs. However, we provide more coverage to fractured systems because in a broader sense, faults are also fractures with displacements. Nelson (1985) in his book classified naturally fractured reservoirs as follows: 


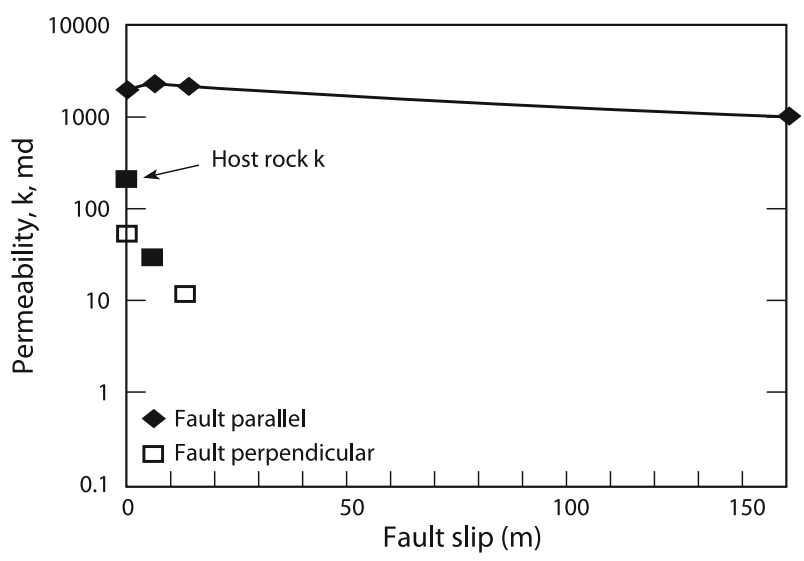

Fig. 2 Fault permeability versus slip, after Flodin et al. (2001) reprinted by permission of SPE

- Type I: Only fractures contribute to reservoir storage capacity (porosity) and conductivity (permeability).

- Type II: Fractures provide overall conductivity (permeability) of the reservoir, and the matrix overall storage capacity (porosity), but the matrix is permeable enough (low permeability) to provide the conductivity for flow from the matrix into fractures.

- Type III: Both fractures and matrix provide conductivity (permeability) of the reservoir but overall storage capacity (porosity) primarily is in the matrix.

- Type IV: Fractures are non-conductive (sealed, filled with minerals, or zero fracture band permeability). Both conductivity (permeability) and storage capacity are provided by the matrix. Such fractures create compartmental reservoirs.

In reality, fractures and faults may occur in many different manners in a given reservoir. When oil-bearing zones with different rock properties are folded together, it is possible that some of the zones may be fractured, faulted, or both, and other zones may not, see Fig. 3. It is also possible that injection and/or migration of warmer or cooler fluids into a stratified formation may create fractures in some zones, or enhance or reduce conductivities of existing ones. These and other geophysical processes may, thus, form reservoirs that consist of both fractured and non-fractured layers commingled together. For instance, many carbonate reservoirs in the Middle East are stratified (layered), and fractures usually occur in tight dolomitic zones. Hence, many of these layered-reservoirs may have some fractured zones with or without crossflow among the layers, depending on stylolitic zones vertical permeabilities and their extent. One of the common reservoir types is a commingled (without crossflow) system with fractured and non-fractured layers due to non-permeable shale or anhydrite zones in between.

Aydin (2000) presented a classification of fractured and faulted reservoirs based on structural discontinuities and geological, geomechanical, and hydraulic characteristics. He also provided a methodology for estimating fracture and fault conductivities and other hydraulic characteristics useful for understanding the transient pressure behavior of fractured and faulted reservoirs. The Aydin (2000) classification for fractured systems is as follows: (1) dilatant-mode fractures/joints, veins, dikes; (2) contraction/compaction-mode fractures/pressure solution seams and compaction bands; (3) shear-mode fractures/faults. Structural and hydraulic characteristics of fundamental elements constituting each of these classes are given in detail in the article. Aydin (2000) further categorized faults as 

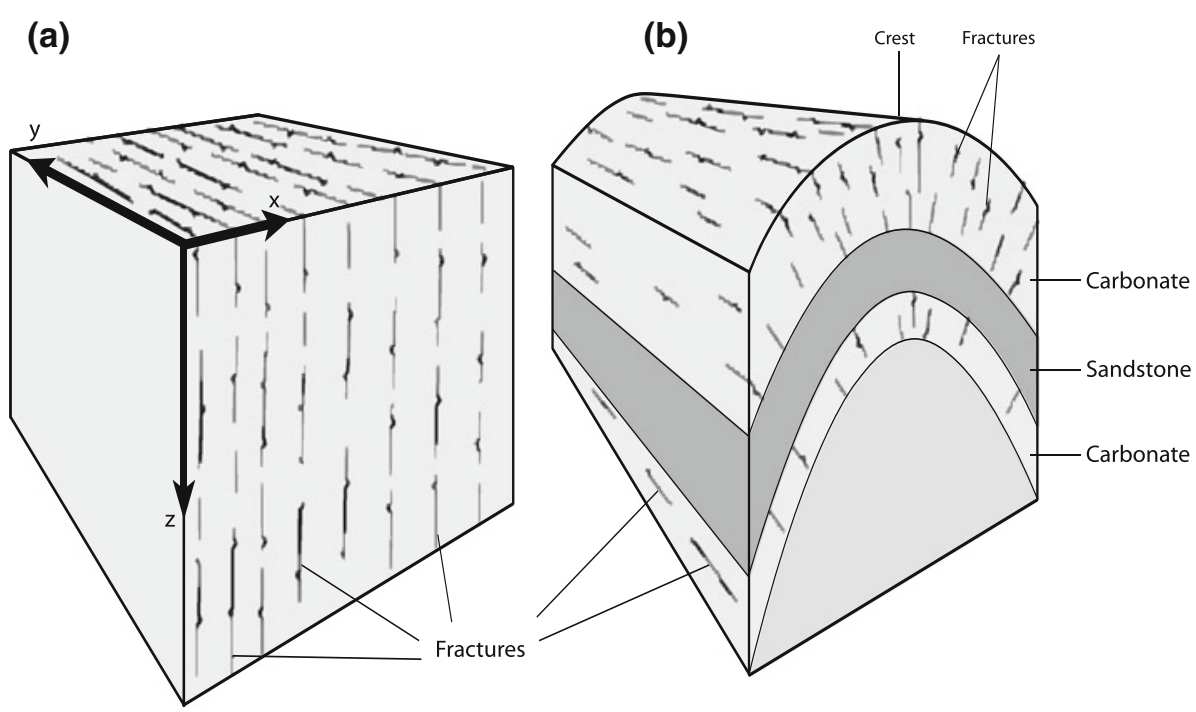

Fig. 3 Schematics of two discretely fractured reservoirs

transmitting faults, sealing faults, vertically transmitting and laterally sealing faults, and partially communicating faults.

Based on the above classifications and overall reservoir point of view (simulations, performance, pressure transient interpretation), the Nelson (1985) classification does not provide any advantages in terms of information about flow in faults, fractures, and the matrix, and particularly, about their conductivities. We therefore divide faulted and fractured reservoirs into four categories as follows:

1. Faults and/or fractures create a network, communicate hydraulically with each other globally, and provide overall conductivity (permeability) of the reservoir, and the matrix provides overall storage capacity (porosity), but they should be permeable enough to provide the conductivity for flow from the matrix into faults and/or fractures. If the matrix permeability is high or similar to the fault and/or fracture permeabilities, or ultra low, the pressure transient behavior of fracture or faulted reservoirs will be similar to the behavior of homogeneous reservoirs. Reservoirs with continuous conductive fracture networks were first modeled as dual-porosity models, as shown in Fig. 4, by Barenblatt et al. (1960) and shortly after by Warren and Root (1963).

2. Faults and/or fractures do not form a continuous conductive network; only a limited number of faults and/or fractures may communicate hydraulically with each other. Faults and/or fractures, and matrix provide conductivity, but overall storage capacity (porosity) is in the matrix. These are usually called discretely fractured reservoirs as shown in Fig. 3. Open and partially sealing faults also create discrete conductive systems. Stratified reservoirs that consist of both fractured and non-fractured layers stocked vertically, as shown in Fig. 3b, should also be called discretely fractured reservoirs. In reservoirs with continuous network of faults and/or fractures, if some faults and/or fractures are mineralized and/or closed, this process also creates discretely fractured reservoirs.

3. The fractures are non-conductive (sealed, filled with minerals, or zero fault or fracture band permeability). Both conductivity (permeability) and storage capacity are provided by the matrix. These reservoirs are referred to as being compartmentalized. 


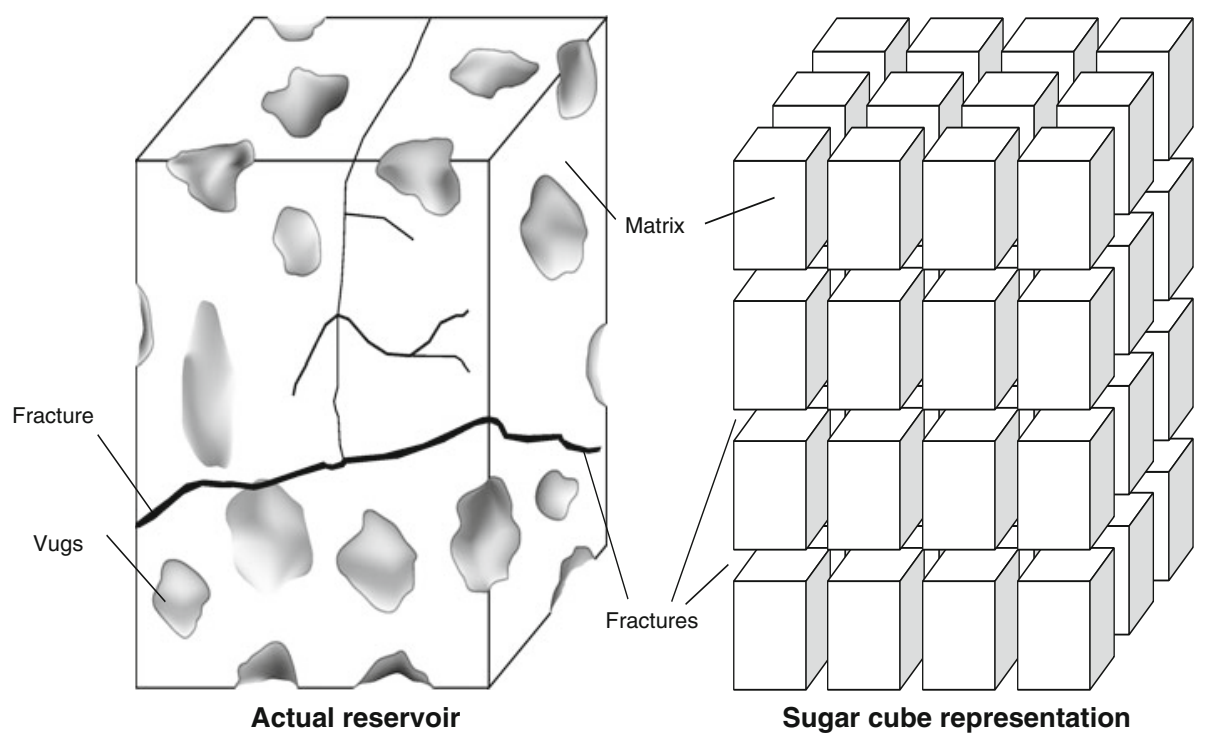

Fig. 4 Warren and Root (1963) dual-porosity reservoir model

4. Only the fractures and/or faults are conductive; the matrix has no permeability and/or porosity. Reservoirs with these fractures are called unconventional fractured basement reservoirs.

The first three types may occur in the same reservoir in different locations depending on the tectonic past of the formation, and other mechanical and chemical processes. Moreover, faults in the fracture reservoirs further complicate the above classification. Therefore, we infrequently observe only one of the Nelson (1985) fractured reservoir types; we usually observe a combination of four types depending on the location of the reservoir structure: crest, flank, etc., layer properties and its geological makeup.

In general, transient pressure behavior of fractured and faulted reservoirs is well studied: a few papers on faulted reservoirs are Horner (1951), Prasad (1975), Cinco-Ley et al. (1976), Yaxley (1978), Tiab and Kumar (1980), Matthai et al. (1998), and Abbaszadeh et al. (2000). Since the study of Barenblatt et al. (1960) more than a few hundred papers have been presented and published on naturally fractured reservoirs using dual-porosity models in the petroleum and groundwater literature [see an extensive list of the literature in Samaniego and Cinco-Ley (2009)].

On the other hand, a very few papers have been published on pressure transient behavior of discretely faulted and fractured systems. Most of these studies focus on the pressure behavior of only one single fracture or fault with finite or infinite conductivity. Most likely, the article by Kuchuk and Habashy (1997) was the first one to be published on the pressure transient behavior of discretely faulted and fractured systems, where multiple faults and fractures with finite and infinite conductivities were considered. These models are applicable to vertical wells completed in an $n$-zone anisotropic medium that contains discrete faults and/or fractures in the $y$ direction. Discrete faults and fractures are bounded above and below by impermeable planes in the $z$ direction and can be finite and/or infinite in the $x$ and $y$ directions. It is shown in this article that discrete faults or fractures dominate the pressure transient behavior of a reservoir for a long time. 


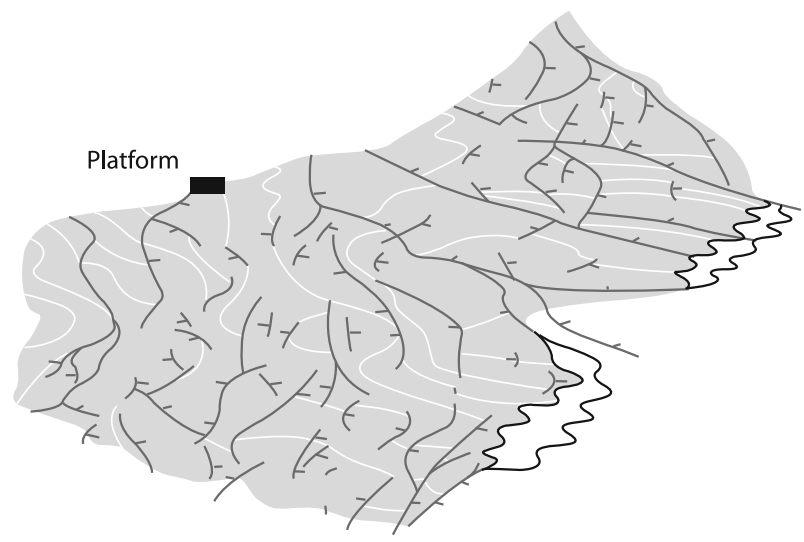

Fig. 5 The fault system in the Cormorant field in North Sea after Ruijtenberg et al. (1990)

The Kuchuk and Habashy (1997) model has captured some of the behavior of pressure transient flow regimes (signatures) of discretely faulted and fractured systems. However, it missed some important flow regimes for discretely faulted and fractured systems, particularly, sparsely and irregularly distributed fractures and/or fault in different directions, like in the Cormorant field in North Sea, as shown in Fig. 5, that is extensively faulted as discussed by Ruijtenberg et al. (1990). As can be seen from this figure, the discrete faults are distributed in $x$ and $y$ directions, and some of them cross each other. Another limitation of the Kuchuk and Habashy (1997) model in a discretely faulted and fractured system is that it does not included those fractures intersecting the wellbore. The conductive wellbore-intersecting fractures normally denominate the pressure behavior of the system during early and middle time periods.

The article by Bogdanov et al. (2003) is also significant to be mentioned here. They have used a three-dimensional single-phase numerical model based on a discrete fracture representation to simulate pressure transient tests in discretely and randomly fractured reservoirs. Their model accurately identified some of the well-know flow regimes of fractured reservoirs.

\section{Mathematical Models for Reservoirs with Discrete Fractures and Faults}

As stated previously, faults and fractures frequently occur in many well-known reservoirs. Since 1960s a considerable amount of work, as referenced above, has been done on faulted and fractured reservoirs, including analytical, semianalytical, and numerical solutions for both finite and infinite fault and fracture conductivities. On the other hand, there are very few pressure transient solutions available for discretely faulted and fractured reservoirs with an arbitrary number and position of conductive faults and/or fractures (Bogdanov et al. 2003). For simplicity, we call reservoirs containing an arbitrary distribution of finite- and/or infinite-conductivity faults and/or fractures discretely fractured network (DFN) or discretely fractured reservoirs. It should be understood that these reservoirs may contain conductive or non-conductive faults and/or fractures, where some of them may intersect each other and/or the wellbore. Characterization of reservoirs with fractures is very important for many areas of reservoir engineering and petroleum geoscience: from drilling to enhanced oil recovery.

Our mesh-free solution technique treats the domain of interest as a whole, rather than discretizing it into small grid blocks as in finite-difference or finite element methods. The 


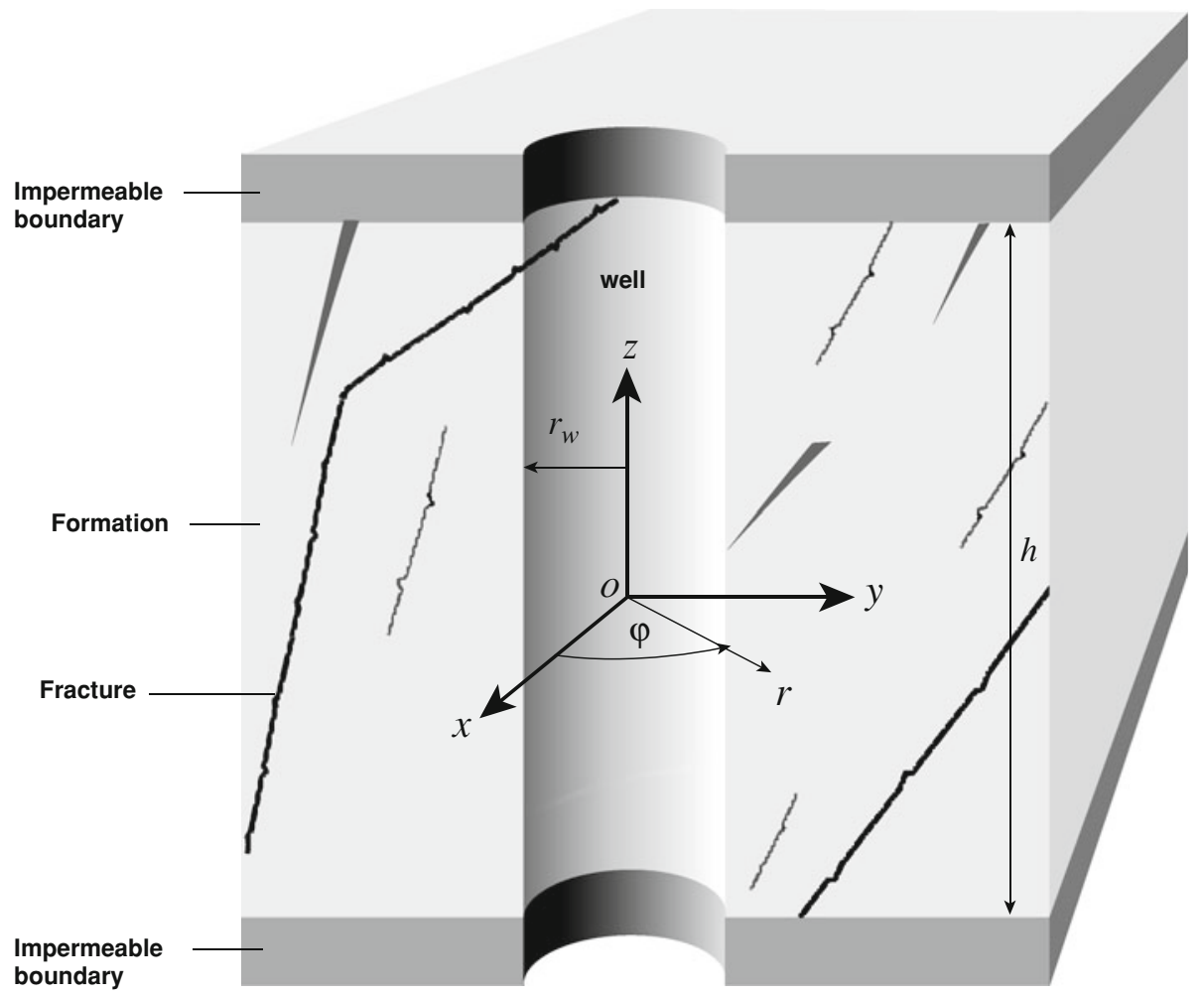

Fig. 6 A schematic of a cylindrical well in a fractured porous medium

mesh-free solutions allow to capture properties and particularities of the transient pressure behavior otherwise inaccessible to mesh-relying methods, as well as to obtain highly accurate solutions. The purpose of this study is to develop a mesh-free pressure transient solution for naturally fractured reservoirs and to present some examples to show their behavior.

Let us consider a single-phase slightly compressible fluid flow in an infinite isotropic reservoir $\Omega \equiv \mathbb{R}^{2}$ produced from one well with a radius $r_{\mathrm{w}}$, as shown in Fig. 6 . The production (flow) rate, $q(t)$, is assumed to be known and time-dependent or constant. The reservoir porosity $\phi$, compressibility $c_{\mathrm{t}}$, horizontal permeability $k$, and fluid viscosity $\mu$ are all assumed to be constant and time and pressure invariant. We also assume that the reservoir contains a network of discrete fractures, where $\Gamma_{i}$ denotes the $i$ th fracture with the half-length $l_{i}$, and $i$ is the fracture counter. In general, as it was said above, some of the fractures may intersect each other. Figures 7 and 8 show a well with an intersecting fracture and two intersecting fractures, respectively. The fluid flow in the matrix outside the fractures is described by the pressure diffusion equation as

$$
\frac{k}{\mu} \frac{\partial^{2} \mathcal{P}}{\partial x^{2}}+\frac{k}{\mu} \frac{\partial^{2} \mathcal{P}}{\partial y^{2}}=\phi c_{\mathrm{t}} \frac{\partial \mathcal{P}}{\partial t},
$$

where $\mathcal{P}=p_{0}-p(x, y, t)$ is a reservoir pressure change induced by fluid withdrawal, $p_{0}$ is the initial reservoir pressure, and $\{x, y\}$ are Cartesian coordinates. In addition, the pressure change $\mathcal{P}$ should also satisfy the following boundary conditions at the wellbore (unless the well intersects a fracture): 


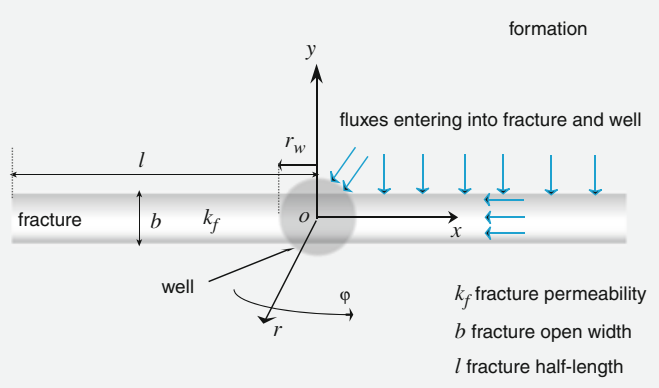

Fig. 7 The schematic of a well with an intersecting fracture in a formation

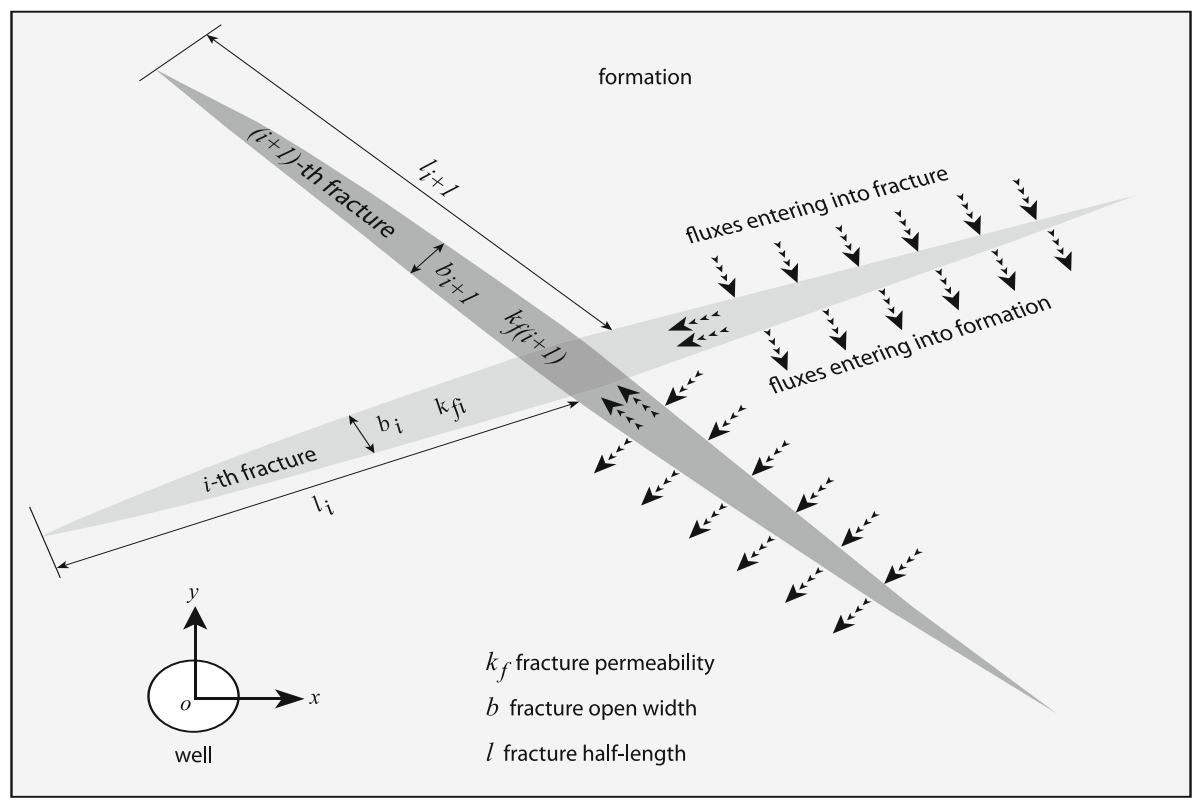

Fig. 8 The schematic of two intersecting fractures in a formation

$$
\begin{aligned}
& \mathcal{P}\left(r_{\mathrm{W}} \cos \varphi, r_{\mathrm{W}} \sin \varphi, t\right)=\mathcal{P}_{\mathrm{W}}(t), \varphi \in[0,2 \pi], \\
& r_{\mathrm{W}} \frac{k}{\mu} \int_{0}^{2 \pi} \frac{\partial \mathcal{P}}{\partial r}\left(r_{\mathrm{W}} \cos \varphi, r_{\mathrm{W}} \sin \varphi, t\right) \mathrm{d} \varphi=-q(t),
\end{aligned}
$$

where $\mathcal{P}_{\mathrm{w}}=p_{0}-p_{\mathrm{w}},\{r, \varphi\}$ denote polar coordinates with origin associated with the wellbore position, as shown in Fig. 6, and $p_{\mathrm{w}}$ is the uniform wellbore pressure over the surface of the cylindrical wellbore (sandface), which is a priori unknown. The fluid flow inside a single fracture $\Gamma_{i}$ can be described by the following equation (Riley et al. 2007): 


$$
\left.\left(\frac{\partial^{2} \mathcal{P}}{\partial x_{i}^{2}}+\frac{1}{F_{i}}\left[\frac{\partial \mathcal{P}}{\partial y_{i}}\right]\right)\right|_{\Gamma_{i}}=0, \frac{\partial \mathcal{P}}{\partial x_{i}}=0 \text { on the extremities of } \Gamma_{i},
$$

where $F_{i}=\frac{k_{\mathrm{f} i} b_{i}}{k}$ is the conductivity of the $i$ th fracture, $k_{\mathrm{f} i}$ is $i$ th fracture permeability, $b_{i}$ is its width, $k$ is the reservoir permeability, $\left\{x_{i}, y_{i}\right\}$ is the local Cartesian coordinate system associated with fracture $\Gamma_{i}$, with $x_{i}$ going in tangential and $y_{i}$ in normal directions to the fracture. $\left[\frac{\partial \mathcal{P}}{\partial y_{i}}\right]=\lim _{y_{i} \rightarrow+0} \frac{\partial \mathcal{P}}{\partial y_{i}}-\lim _{y_{i} \rightarrow-0} \frac{\partial \mathcal{P}}{\partial y_{i}}$ denotes a normal pressure derivative jump across the fracture; i.e., a difference of normal pressure derivative values at both sides of the fracture. The integration of Eq. 4 over the fracture length, together with the boundary conditions on the extremities, gives the boundary condition for the incompressible fluid flow inside the fracture, which can be expressed as

$$
\int_{-l_{i}}^{l_{i}}\left[\frac{\partial \mathcal{P}}{\partial y_{i}}\right] \mathrm{d} x_{i}=0
$$

For an infinite-conductivity fracture, the governing equation (Eq. 4) can be simplified by tending the fracture conductivity $F_{i}$ to infinity, which together with conditions on fracture extremities yields

$$
\frac{\partial \mathcal{P}}{\partial x_{i}}=0 \text { on } \Gamma_{i}
$$

or

$$
\mathcal{P}=\mathcal{P}_{\mathrm{f} i}(t) \text { on } \Gamma_{i},
$$

where $\mathcal{P}_{\mathrm{f} i}=p_{0}-p_{\mathrm{f} i}$ and $\mathcal{P}_{\mathrm{f} i}$ is a uniform pressure induced on a fracture face $\Gamma_{i}$, which is a priori unknown (as with the wellbore case). Finite-conductivity fractures will be treated below in a separate section.

Now let us assume that some of the fractures intersect each other and/or the wellbore. Let $I_{j}$ denote a set of indices $i$ belonging to a $j$ th group of connected fractures (including a wellbore), and let $j=0$ correspond to a group containing the well and $\mathcal{P}_{\mathrm{f} 0}=\mathcal{P}_{\mathrm{w}}$. In this case, the conditions given by Eqs. 3, 4, 6, and 7 should be reformulated as follows:

$$
\begin{aligned}
& \mathcal{P}=\mathcal{P}_{\mathrm{f} 0}=\mathcal{P}_{\mathrm{w}} \text { on } \Gamma_{i}, \quad i \in I_{0}, \\
& r_{\mathrm{w}} \frac{k}{\mu} \int_{0}^{2 \pi} \frac{\partial \mathcal{P}}{\partial r}\left(r_{\mathrm{w}} \cos \varphi, r_{\mathrm{w}} \sin \varphi, t\right) \mathrm{d} \varphi+\frac{k}{\mu} \sum_{i \in I_{0}} \int_{-l_{i}}^{l_{i}}\left[\frac{\partial \mathcal{P}}{\partial y_{i}}\right] \mathrm{d} x_{i}=-q(t), \\
& \mathcal{P}=\mathcal{P}_{\mathrm{f} j} \text { on } \Gamma_{i}, \quad i \in I_{j}, \quad j>0, \\
& \frac{k}{\mu} \sum_{i \in I_{j}} \int_{-l_{i}}^{l_{i}}\left[\frac{\partial \mathcal{P}}{\partial y_{i}}\right] \mathrm{d} x_{i}=0, \quad j>0 .
\end{aligned}
$$

Note that physically these equations correspond to the equality of the pressure change within a connected group (since fractures and wellbore are assumed to have an infinite conductivity, i.e., to be under the uniform pressure condition) and to the condition on the total flow rate within a group - which should be $q(t)$ if the well is present in the group, and 0 otherwise. To close the system, we should also add the initial condition

$$
\mathcal{P}(x, y, 0)=0,
$$


corresponding to zero pressure change at $t=0$. Let us define to the following dimensionless variables to simplify the above equations:

$$
\begin{aligned}
& t_{\mathrm{D}}=\frac{k t}{\mu c_{\mathrm{t}} \phi l^{2}}, p_{\mathrm{D}}=\frac{2 k \pi h}{\mu q_{0}} \mathcal{P}, x_{\mathrm{D}}=\frac{x}{l}, y_{\mathrm{D}}=\frac{y}{l}, \\
& l_{\mathrm{D} i}=\frac{l_{i}}{l}, x_{\mathrm{D} i}=\frac{x_{i}}{l_{i}}, y_{\mathrm{D} i}=\frac{y_{i}}{l_{i}}, r_{\mathrm{D}}=\frac{r}{l}, r_{\mathrm{Dw}}=\frac{r_{\mathrm{w}}}{l},
\end{aligned}
$$

where $l$ denotes a reference length (half-length of the longest fracture, for example), $h$ is the reservoir height, and $q_{0}$ is a reference flow rate. In terms of these dimensionless variables, the mathematical model defined by Eqs. 1, and 8-12 can be rewritten as

$$
\begin{aligned}
& \frac{\partial^{2} p_{\mathrm{D}}}{\partial x_{\mathrm{D}}^{2}}+\frac{\partial^{2} p_{\mathrm{D}}}{\partial y_{\mathrm{D}}^{2}}=\frac{\partial p_{\mathrm{D}}}{\partial t_{\mathrm{D}}}, \\
& p_{\mathrm{D}}=p_{\mathrm{Df} 0}\left(t_{\mathrm{D}}\right)=p_{\mathrm{Dw}}\left(t_{\mathrm{D}}\right) \text { on } \Gamma_{i}, \quad i \in I_{0}, \\
& \frac{r_{\mathrm{Dw}}}{2 \pi} \int_{0}^{2 \pi} \frac{\partial p_{\mathrm{D}}}{\partial r_{\mathrm{D}}}\left(r_{\mathrm{Dw}} \cos \varphi, r_{\mathrm{Dw}} \sin \varphi, t_{\mathrm{D}}\right) \mathrm{d} \varphi+\frac{1}{2 \pi} \sum_{i \in I_{0}} \int_{-1}^{1}\left[\frac{\partial p_{\mathrm{D}}}{\partial y_{\mathrm{D} i}}\right] \mathrm{d} x_{\mathrm{D} i}=-q_{\mathrm{D}}(t), \\
& p_{\mathrm{D}}=p_{\mathrm{Df} j}\left(t_{\mathrm{D}}\right) \text { on } \Gamma_{i}, i \in I_{j}, \quad j>0, \\
& \frac{1}{2 \pi} \sum_{i \in I_{j}} \int_{-1}^{1}\left[\frac{\partial p_{\mathrm{D}}}{\partial y_{\mathrm{D} i}}\right] \mathrm{d} x_{\mathrm{D} i}=0, \quad j>0, \\
& p_{\mathrm{D}}\left(x_{\mathrm{D}}, y_{\mathrm{D}}, 0\right)=0 .
\end{aligned}
$$

Application of the Laplace transform to this system yields

$$
\begin{aligned}
& \frac{\partial^{2} \bar{p}_{\mathrm{D}}}{\partial x_{\mathrm{D}}^{2}}+\frac{\partial^{2} \bar{p}_{\mathrm{D}}}{\partial y_{\mathrm{D}}^{2}}=s \bar{p}_{\mathrm{D}}, \\
& \bar{p}_{\mathrm{D}}=\bar{p}_{\mathrm{Df} 0}(s)=\bar{p}_{\mathrm{Dw}}(s) \text { on } \Gamma_{i}, \quad i \in I_{0}, \\
& \frac{r_{\mathrm{Dw}}}{2 \pi} \int_{0}^{2 \pi} \frac{\partial \bar{p}_{\mathrm{D}}}{\partial r_{\mathrm{D}}}\left(r_{\mathrm{Dw}} \cos \varphi, r_{\mathrm{Dw}} \sin \varphi, s\right) \mathrm{d} \varphi+\frac{1}{2 \pi} \sum_{i \in I_{0}} \int_{-1}^{1}\left[\frac{\partial \bar{p}_{\mathrm{D}}}{\partial y_{\mathrm{D} i}}\right] \mathrm{d} x_{\mathrm{D} i}=-\bar{q}_{\mathrm{D}}(s), \\
& \bar{p}_{\mathrm{D}}=\bar{p}_{\mathrm{Df} j}(s) \text { on } \Gamma_{i}, \quad i \in I_{j}, \quad j>0, \\
& \frac{1}{2 \pi} \sum_{i \in I_{j}} \int_{-1}^{1}\left[\frac{\partial \bar{p}_{\mathrm{D}}}{\partial y_{\mathrm{D} i}}\right] \mathrm{d} x_{\mathrm{D} i}=0, \quad j>0 .
\end{aligned}
$$

\subsection{Transient Solution for Infinite-Conductivity Fractures}

Let us assume that the dimensionless pressure change "induced by the wellbore" can be expanded into the Fourier series as

$$
\begin{aligned}
& \bar{p}_{\mathrm{D} 0}\left(r_{\mathrm{D}} \cos \varphi, r_{\mathrm{D}} \sin \varphi, s\right)=\bar{a}_{0}(s) \frac{\mathrm{K}_{0}\left(\sqrt{s} r_{\mathrm{D}}\right)}{\mathrm{K}_{1}\left(\sqrt{s} r_{\mathrm{Dw}}\right) \sqrt{s} r_{\mathrm{Dw}}} \\
& +\sum_{n=1}^{\infty} \frac{2 \mathrm{~K}_{n}\left(\sqrt{s} r_{\mathrm{D}}\right)}{\left[\mathrm{K}_{n+1}\left(\sqrt{s} r_{\mathrm{Dw}}\right)+\mathrm{K}_{n-1}\left(\sqrt{s} r_{\mathrm{Dw}}\right)\right] \sqrt{s} r_{\mathrm{Dw}}}\left(\bar{a}_{n}(s) \cos n \varphi+\bar{b}_{n}(s) \sin n \varphi\right),
\end{aligned}
$$


where $\mathrm{K}_{n}$ denotes a Modified Bessel function of the second kind of $n$th order. Similarly, we assume that the pressure change "induced by the $i$ th fracture" can be represented in its local coordinate system in the following way:

$$
\begin{aligned}
\bar{p}_{\mathrm{D} i}\left(x_{\mathrm{D} i}, y_{\mathrm{D} i}, s\right) & =\frac{1}{\pi} \sum_{n=0}^{\infty} \bar{c}_{i n}(s) \int_{-1}^{1} \mathrm{~K}_{0}\left(\sqrt{s} l_{\mathrm{D} i} \sqrt{\left(x_{\mathrm{D} i}-\xi\right)^{2}+y_{\mathrm{D} i}^{2}}\right) \frac{T_{n}(\xi)}{\sqrt{1-\xi^{2}}} \mathrm{~d} \xi \\
& =\frac{1}{\pi} \sum_{n=0}^{\infty} \bar{c}_{i n}(s) \int_{0}^{\pi} \mathrm{K}_{0}\left(\sqrt{s} l_{\mathrm{D} i} \sqrt{\left(x_{\mathrm{D} i}-\cos \psi\right)^{2}+y_{\mathrm{D} i}^{2}}\right) \cos n \psi \mathrm{d} \psi .
\end{aligned}
$$

It is easy to see that the above function definitions automatically satisfy Eq. 20. Generally speaking, the function defined by Eq. 25 corresponds to a solution of the diffusion equation in an infinite reservoir with the flux density specified on the wellbore as

$$
\bar{q}_{\mathrm{Dw}}(s, \varphi)=\bar{a}_{0}(s)+\sum_{n=1}^{\infty}\left[\bar{a}_{n}(s) \cos n \varphi+\bar{b}_{n}(s) \sin n \varphi\right],
$$

while Eq. 26 defines a plane-source solution with flux density distribution on the fracture $\Gamma_{i}$ equal to

$$
\bar{q}_{\mathrm{Df} i}\left(s, x_{\mathrm{D} i}\right)=\frac{1}{2}\left[\frac{\partial \bar{p}_{\mathrm{D}}}{\partial y_{\mathrm{D} i}}\right]=\sum_{n=0}^{\infty} \bar{c}_{i n}(s) \frac{T_{n}\left(x_{\mathrm{D} i}\right)}{\sqrt{1-x_{\mathrm{D} i}^{2}}} .
$$

Notice that instead of Eq. 26 we could also use Mathieu functions, but since their computation is difficult and time consuming, the alternative is preferable. On the other hand, the functions defined by Eq. 26 can be easily evaluated, as shown in Appendix. Another reason to use these functions over others is that they reflect the primary singularity of the flux density-its divergence on the edges of the fracture, which insures fast convergence of the corresponding series.

The unknown expansion coefficients $\bar{a}_{n}(s), \bar{b}_{n}(s)$, and $\bar{c}_{i n}(s)$ can be found from Eqs. 21-24. That is, if we denote

$$
\begin{aligned}
& \bar{p}_{\mathrm{D} 0 n 1}\left[x_{\mathrm{D}}\left(r_{\mathrm{D}}, \varphi\right), y_{\mathrm{D}}\left(r_{\mathrm{D}}, \varphi\right)\right]=\frac{2 \mathrm{~K}_{n}\left(\sqrt{s} r_{\mathrm{D}}\right)}{\left[\mathrm{K}_{n+1}\left(\sqrt{s} r_{\mathrm{Dw}}\right)+\mathrm{K}_{n-1}\left(\sqrt{s} r_{\mathrm{Dw}}\right)\right] \sqrt{s} r_{\mathrm{Dw}}} \cos n \varphi, \\
& \bar{p}_{\mathrm{D} 0 n 2}\left[x_{\mathrm{D}}\left(r_{\mathrm{D}}, \varphi\right), y_{\mathrm{D}}\left(r_{\mathrm{D}}, \varphi\right)\right]=\frac{2 \mathrm{~K}_{n}\left(\sqrt{s} r_{\mathrm{D}}\right)}{\left[\mathrm{K}_{n+1}\left(\sqrt{s} r_{\mathrm{Dw}}\right)+\mathrm{K}_{n-1}\left(\sqrt{s} r_{\mathrm{Dw}}\right)\right] \sqrt{s} r_{\mathrm{Dw}}} \sin n \varphi, \\
& \bar{p}_{\mathrm{Din}}\left[x_{\mathrm{D}}\left(x_{\mathrm{D} i}, y_{\mathrm{D} i}\right), y_{\mathrm{D}}\left(x_{\mathrm{D} i}, y_{\mathrm{D} i}\right)\right]=\frac{1}{\pi} \int_{0}^{\pi} \mathrm{K}_{0}\left[\sqrt{s} l_{\mathrm{D} i} \sqrt{\left(x_{\mathrm{D} i}-\cos \psi\right)^{2}+y_{\mathrm{D} i}^{2}}\right] \cos n \psi \mathrm{d} \psi,
\end{aligned}
$$

- notice that here we omit the coordinate transformation from a local coordinate system to a global one $\left(x_{\mathrm{D}}, y_{\mathrm{D}}\right) \leftrightarrow\left(x_{\mathrm{D} i}, y_{\mathrm{D} i}\right)$, which is trivial but cumbersome-Eqs. 21-24 can then be rewritten as follows: 


$$
\begin{aligned}
& \sum_{n=0}^{\infty} \bar{a}_{n} \bar{p}_{\mathrm{D} 0 n 1}\left[x_{\mathrm{D}}\left(r_{\mathrm{D}}, \varphi\right), y_{\mathrm{D}}\left(r_{\mathrm{D}}, \varphi\right)\right]+\sum_{n=1}^{\infty} \bar{b}_{n} \bar{p}_{\mathrm{D} 0 n 2}\left[x_{\mathrm{D}}\left(r_{\mathrm{D}}, \varphi\right), y_{\mathrm{D}}\left(r_{\mathrm{D}}, \varphi\right)\right] \\
& +\sum_{k=1}^{M} \sum_{n=0}^{\infty} \bar{c}_{k n} \bar{p}_{\mathrm{D} k n}\left[x_{\mathrm{D}}\left(x_{\mathrm{D} k}, y_{\mathrm{D} k}\right), y_{\mathrm{D}}\left(x_{\mathrm{D} k}, y_{\mathrm{D} k}\right)\right]=\bar{p}_{\mathrm{Dw}}(s) \text { on } \Gamma_{i}, \quad i \in I_{0}, \\
& \bar{a}_{0}+\sum_{i \in I_{0}} \bar{c}_{i 0}=\bar{q}_{\mathrm{D}}(s), \\
& \sum_{n=0}^{\infty} \bar{a}_{n} \bar{p}_{\mathrm{D} 0 n 1}\left[x_{\mathrm{D}}\left(r_{\mathrm{D}}, \varphi\right), y_{\mathrm{D}}\left(r_{\mathrm{D}}, \varphi\right)\right]+\sum_{n=1}^{\infty} \bar{b}_{n} \bar{p}_{\mathrm{D} 0 n 2}\left[x_{\mathrm{D}}\left(r_{\mathrm{D}}, \varphi\right), y_{\mathrm{D}}\left(r_{\mathrm{D}}, \varphi\right)\right] \\
& +\sum_{k=1}^{M} \sum_{n=0}^{\infty} \bar{c}_{k n} \bar{p}_{\mathrm{D} k n}\left[x_{\mathrm{D}}\left(x_{\mathrm{D} k}, y_{\mathrm{D} k}\right), y_{\mathrm{D}}\left(x_{\mathrm{D} k}, y_{\mathrm{D} k}\right)\right]=\bar{p}_{\mathrm{Df} j}(s) \text { on } \Gamma_{i}, \quad i \in I_{j}, j>0 \\
& \sum_{i \in I_{j}} \bar{c}_{i 0}=0, \quad j>0,
\end{aligned}
$$

where $M$ denotes the number of fractures in the reservoir.

Now, to solve this system we must restrict ourselves to a finite number of terms in each of the series defined by Eqs. 25, 26. We suggest taking $N \approx 10$ to 30 elements for every series. For very long fractures it might be wise to divide them into a few smaller ones connected at the endpoints, to improve numerical stability. The next step is the discretization of Eqs. 32-35. It is also possible to expand the equations over some basis functions like $\cos (n \varphi)$ and $\sin (n \varphi)$ on the wellbore, and $T_{k}\left(x_{\mathrm{D} i}\right)$ (Chebyshev polynomials) on the fractures; however, this would be very time consuming. By considering Eqs. 32-35 only on special grid nodes, such as $\varphi_{m}=\frac{\pi}{N}(0.5+2 m), m=0,1, \ldots, N-1$, and $x_{\operatorname{Dim}}=\cos \left[\frac{\pi}{N}(0.5+m)\right], m=0,1, \ldots$, $N-1$, we can attain the same result in less time as this procedure corresponds to such an expansion in the sense of Gaussian quadrature rules. One can choose the number of nodes to be greater than the number of coefficients, and thus instead of searching for the exact solution of the discretized system, compute its pseudo-solution in the least squares sense. However, this approach did not prove to give any advantage.

\subsection{Transient Solutions for Finite-Conductivity Fractures}

In this section, we show how the previous method can be extended to take into account finite-conductivity fractures. As we have already stated, the flow inside a fracture in its local coordinate system is described by the following equation:

$$
\left.\left(\frac{\partial^{2} \mathcal{P}}{\partial x_{i}^{2}}+\frac{1}{F_{i}}\left[\frac{\partial \mathcal{P}}{\partial y_{i}}\right]\right)\right|_{\Gamma_{i}}=0,
$$

which can be rewritten in Laplace domain in terms of dimensionless variables defined by Eq. 13 as

$$
\left.\left(\frac{\partial^{2} \bar{p}_{\mathrm{D}}}{\partial x_{\mathrm{D} i}^{2}}+\frac{l_{\mathrm{D} i}}{F_{\mathrm{D} i}}\left[\frac{\partial \bar{p}_{\mathrm{D}}}{\partial y_{\mathrm{D} i}}\right]\right)\right|_{\Gamma_{i}}=0,
$$


where

$$
F_{\mathrm{D} i}=\frac{k_{\mathrm{f} i} b_{i}}{k l} .
$$

Equation 37 is only valid for an isolated fracture. In the case of intersecting fractures, it must include additional terms corresponding to the flow exchange in the intersection point. Suppose that the fracture $i$ intersects the fractures $i_{1}, i_{2}, \ldots, i_{N_{i}}$ at the points $x_{\mathrm{D} i i_{1}}, x_{\mathrm{D} i i_{2}}, \ldots, x_{\mathrm{D} i i_{N_{i}}}$, respectively. Equation 37 should then be replaced with the following:

$$
\left.\left\{\frac{\partial^{2} \bar{p}_{\mathrm{D}}}{\partial x_{\mathrm{D} i}^{2}}+\frac{l_{\mathrm{D} i}}{F_{\mathrm{D} i}}\left(\left[\frac{\partial \bar{p}_{\mathrm{D}}}{\partial y_{\mathrm{D} i}}\right]-2 \pi \sum_{k=1}^{N_{i}} \bar{q}_{i i_{k}} \delta\left(x_{\mathrm{D} i}-x_{\mathrm{D} i i_{k}}\right)\right)\right\}\right|_{\Gamma_{i}}=0,
$$

where $q_{i i_{k}}$ corresponds to the flux passing to $i$ th fracture from $i_{k}$ th in the corresponding intersection point and $\delta$ is the Dirac delta functional. It is evident that due to the continuity of the flux $\bar{q}_{i i_{k}}=-\bar{q}_{i_{k} i}$.

Assuming that the pressure change generated by $i$ th fracture can still be expressed by Eq. 26, we then can rewrite Eq. 39 as

$$
\begin{aligned}
\bar{p}_{\mathrm{D}}\left(l_{\mathrm{D} i} \cos \varphi_{i}\right)= & \frac{l_{\mathrm{D} i}}{F_{\mathrm{D} i}}\left\{2 \bar{c}_{i 0}\left(\sin \varphi_{i}-\varphi_{i} \cos \varphi_{i}\right)+\bar{c}_{i 1}\left[\varphi_{i}-\frac{1}{2} \sin \left(2 \varphi_{i}\right)\right]\right. \\
& +\sum_{n=2}^{\infty} \frac{\bar{c}_{i n}}{n}\left\{\frac{\sin \left[(n-1) \varphi_{i}\right]}{n-1}+\frac{\sin \left[(n+1) \varphi_{i}\right]}{n+1}\right\} \\
& \left.-2 \pi \sum_{k=1}^{N_{i}} \bar{q}_{i i_{k}} \mathrm{H}\left(\varphi_{i i_{k}}-\varphi_{i}\right)\left(\cos \varphi_{i}-\cos \varphi_{i i_{k}}\right)\right\}+\bar{A}_{i},
\end{aligned}
$$

where $x_{\mathrm{D} i}=l_{\mathrm{D} i} \cos \varphi_{i}, \varphi_{i} \in[0, \pi]$, and $\mathrm{H}$ is the Heaviside step function, defined as

$$
\mathrm{H}(x)= \begin{cases}0, & \text { if } x \leq 0 \\ 1, & \text { otherwise }\end{cases}
$$

It implies that the right-hand side of Eq. 40 should replace the right-hand sides of Eqs. 34 and 32 (for fractures only). The unknowns $\bar{q}_{i i_{k}}$ and $\bar{A}_{i}$ can be found from the pressure continuity condition, given as

$$
\left.\bar{p}_{\mathrm{D}}\left(l_{\mathrm{D} i} \cos \varphi_{i i_{k}}\right)\right|_{\Gamma_{i}}=\left.\bar{p}_{\mathrm{D}}\left(l_{\mathrm{D} i_{k}} \cos \varphi_{i_{k} i}\right)\right|_{\Gamma_{i_{k}}},
$$

stating that the right-hand sides of Eq. 40 for intersecting fractures should be equal at the intersection point, and the condition on the total flux passing through the fracture, which is

$$
\bar{c}_{i 0}+\sum_{k=1}^{N_{i}} \bar{q}_{i i_{k}}=0
$$

Note that if $i=0$ (i.e., the "fracture" is actually the wellbore), then these conditions can be expressed as

$$
\begin{aligned}
& \bar{p}_{\mathrm{Dw}}=\left.\bar{p}_{\mathrm{D}}\left(l_{\mathrm{D} i_{k}} \cos \varphi_{i_{k} 0}\right)\right|_{\Gamma_{i_{k}}}, \\
& \bar{a}_{0}+\sum_{k=1}^{N_{0}} \bar{q}_{0 i_{k}}=\bar{q}_{\mathrm{D}}(s) .
\end{aligned}
$$




\subsection{Compressible Fluid Flow Inside Finite-Conductivity Fractures}

In most of the finite-conductivity fracture solutions by Riley et al. (2007), Cinco-Ley and Samaniego (1981), Cinco-Ley et al. (1978), and Lee and Brockenbrough (1986), but not in the solutions by Kuchuk and Habashy (1997), the fluid flow within the fracture is assumed to be incompressible. In this section, we explain how the solution should be modified to take into account compressible flow inside finite-conductivity fractures. Let us assign a porosity $\phi_{\mathrm{f} i}$ and total compressibility $c_{\mathrm{tf} i}$ to the fracture $\Gamma_{i}$. The governing equation for the flow inside the fracture (it is assumed that Darcy's law is valid) then becomes

$$
\phi_{\mathrm{f} i} c_{\mathrm{tf} i} \frac{\partial \mathcal{P}}{\partial t}=\frac{k_{\mathrm{f} i}}{\mu} \frac{\partial^{2} \mathcal{P}}{\partial x_{i}^{2}}+\left.\frac{k}{\mu}\left[\frac{\partial \mathcal{P}}{\partial y_{i}}\right]\right|_{\Gamma_{i}}=0,
$$

which we can write in Laplace domain in dimensionless form as

$$
\left(\chi_{\mathrm{f} i} l_{\mathrm{D} i}^{2}\right) s \bar{p}_{\mathrm{D}}=\frac{\partial^{2} \bar{p}_{\mathrm{D}}}{\partial x_{\mathrm{D} i}^{2}}+\left.\frac{l_{\mathrm{D} i}}{F_{\mathrm{D} i}}\left[\frac{\partial \bar{p}_{\mathrm{D}}}{\partial y_{\mathrm{D} i}}\right]\right|_{\Gamma_{i}},
$$

where

$$
\chi_{\mathrm{f} i}=\frac{c_{\mathrm{tf} i} \phi_{\mathrm{f} i}}{c_{\mathrm{t}} \phi} \times \frac{k}{k_{\mathrm{f} i}}
$$

is the matrix-fracture diffusivity ratio. Again Eq. 47 is valid only for an isolated fracture. If the fracture intersects other fractures and/or the wellbore, the governing equation takes the following form:

$$
\left(\chi_{\mathrm{f} i} l_{\mathrm{D} i}^{2}\right) s \bar{p}_{\mathrm{D} i}=\frac{\partial^{2} \bar{p}_{\mathrm{D}}}{\partial x_{\mathrm{D} i}^{2}}+\left.\frac{l_{\mathrm{D} i}}{F_{\mathrm{D} i}}\left(\left[\frac{\partial \bar{p}_{\mathrm{D}}}{\partial y_{\mathrm{D} i}}\right]-2 \pi \sum_{k=1}^{N_{i}} \bar{q}_{i i_{k}} \delta\left(x_{\mathrm{D} i}-x_{\mathrm{D} i i_{k}}\right)\right)\right|_{\Gamma_{i}} .
$$

Now using the expression for $\left[\frac{\partial \bar{p}_{\mathrm{D}}}{\partial y_{\mathrm{D} i}}\right]$ from Eq. 28 , we can solve Eq. 49 for $\bar{p}_{\mathrm{D}}$ :

$$
\bar{p}_{\mathrm{D}}\left(x_{\mathrm{D} i}\right)=\frac{2 l_{\mathrm{D} i}}{F_{\mathrm{D} i}}\left[\sum_{n=0}^{\infty} \bar{c}_{i n}(s) \bar{\Theta}_{n}\left(\chi_{\mathrm{f} i} l_{\mathrm{D} i}^{2} s, x_{\mathrm{D} i}\right)-\pi \sum_{k=1}^{N_{i}} \bar{q}_{i i_{k}} \bar{\Psi}\left(\chi_{\mathrm{f} i} l_{\mathrm{D} i}^{2} s, x_{\mathrm{D} i}, x_{\mathrm{D} i k}\right)\right],
$$

where

$$
\begin{aligned}
& \bar{\Theta}_{2 m}\left(\chi_{\mathrm{f} i} l_{\mathrm{D} i}^{2} s, x_{\mathrm{D} i}\right)=(-1)^{m} \pi \sum_{r=0}^{\infty} \frac{\mathrm{J}_{2 m}(\pi r) \cos \left(\pi r x_{\mathrm{D} i}\right)}{\chi_{\mathrm{f} i} l_{\mathrm{D} i}^{2} s+\pi^{2} r^{2}}\left(1-\frac{\delta_{0 r}}{2}\right) \\
& \bar{\Theta}_{2 m+1}\left(\chi_{\mathrm{f} i} l_{\mathrm{D} i}^{2} s, x_{\mathrm{D} i}\right)=(-1)^{m} \pi \sum_{r=0}^{\infty} \frac{\mathrm{J}_{2 m+1}[\pi(r+0.5)] \cos \left[\pi(r+0.5) x_{\mathrm{D} i}\right]}{\chi_{\mathrm{f} i} l_{\mathrm{D} i}^{2} s+\pi^{2}(r+0.5)^{2}},
\end{aligned}
$$

and

$$
\bar{\Psi}\left(\chi_{\mathrm{f} i} l_{\mathrm{D} i}^{2} s, x_{\mathrm{D} i}, x_{\mathrm{D} i k}\right)=\frac{1}{2 \chi_{\mathrm{f} i} l_{\mathrm{D} i}^{2} s}+\sum_{r=1}^{\infty} \frac{\cos \left[\frac{\pi r}{2}\left(x_{\mathrm{D} i}+1\right)\right] \cos \left[\frac{\pi r}{2}\left(x_{\mathrm{D} i i_{k}}+1\right)\right]}{\chi_{\mathrm{f} i} l_{\mathrm{D} i}^{2} s+\pi^{2} r^{2}} .
$$

Using the tables of series sums from Prudnikov et al. (1981), we can reduce the last expression to 


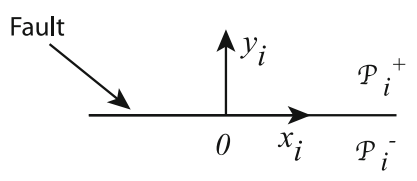

Fig. 9 A schematic of a fault

$$
\begin{aligned}
\bar{\Psi}\left(\chi_{\mathrm{f} i} l_{\mathrm{D} i}^{2} s, x_{\mathrm{D} i}, x_{\mathrm{D} i k}\right)= & \frac{\pi}{4 \chi_{\mathrm{f} i} l_{\mathrm{D} i}^{2} s} \\
& \times \frac{\operatorname{ch}\left[\frac{\chi_{\mathrm{f} i} l_{\mathrm{D} i}^{2} s}{2 \pi}\left(x_{\mathrm{D} i}+x_{\mathrm{D} i i_{k}}\right)\right]+\operatorname{ch}\left[\frac{\chi_{\mathrm{fi}} l_{\mathrm{D} i}^{2} s}{2 \pi}\left(2-\left|x_{\mathrm{D} i}-x_{\mathrm{D} i i_{k}}\right|\right)\right]}{\operatorname{sh}\left(\chi_{\mathrm{f} i} l_{\mathrm{D} i}^{2} s / \pi\right)} .
\end{aligned}
$$

The continuity conditions given by Eqs. 42-45 at fracture intersection points stay the same. If the fracture and formation storativity are of the same order of magnitude, the matrix-fracture diffusivity ratio — as a proportionality but not strictly speaking — is given as

$$
\chi_{\mathrm{f} i} \sim \frac{k}{k_{\mathrm{f} i}}=\frac{1}{F_{\mathrm{D} i}} \frac{b}{l},
$$

which is generally very small, and thus the compressibility effects of the fracture fluid can be neglected in most cases.

\subsection{Transient Solutions for Reservoirs with Sealing and Leaky Faults}

In this section, we will explain how non- or low-conductive faults can be incorporated in fractured reservoir model presented above. The flow of a single-phase slightly compressible fluid through a fault can be described by the following equation (see Fig. 9):

$$
\frac{k}{\mu} \frac{\partial \mathcal{P}^{+}}{\partial y_{i}}=\frac{k}{\mu} \frac{\partial \mathcal{P}^{-}}{\partial y_{i}}=\alpha_{i}\left(\mathcal{P}^{+}-\mathcal{P}^{-}\right) \text {on the } i \text { th fault, }
$$

where $\mathcal{P}^{+}$and $\mathcal{P}^{-}$are the pressure values on the different sides of the fault and

$$
\alpha_{i}=\frac{k_{\mathrm{f}_{i}}}{\mu} \frac{1}{b_{i}}
$$

is the fault transmissibility (Kuchuk and Habashy 1997; Yaxley 1978), $\left\{x_{i}, y_{i}\right\}$ are the coordinates associated with the fault with the origin in its middle and $x_{i}$ axis being parallel to the fault.

Applying the Laplace transform and using dimensionless variables introduced earlier we can rewrite Eq. 56 as

$$
\frac{\partial \bar{p}_{\mathrm{D}}^{+}}{\partial y_{\mathrm{D} i}}=\frac{\partial \bar{p}_{\mathrm{D}}^{-}}{\partial y_{\mathrm{D} i}}=\alpha_{\mathrm{D} i}\left(\bar{p}_{\mathrm{D}}^{+}-\bar{p}_{\mathrm{D}}^{-}\right), \quad-1<x_{\mathrm{D} i}<1,
$$

where

$$
\alpha_{\mathrm{D} i}=\frac{k_{\mathrm{f}_{i}}}{k} \frac{l_{i}}{b_{i}}
$$

is the dimensionless fault transmissibility, and $l_{i}$ is the fault half-length.

Similarly to the fracture case, we need to find a "suitable" function for representing the pressure change induced by this fault. According to Eq. 58, it should be discontinuous on 
the fault, have a "continuous" generalized normal derivative on it (i.e., a true derivative having a removable discontinuity) and also be continuous everywhere else. One of the possible functions satisfying these properties is

$$
\begin{aligned}
\bar{p}_{\mathrm{D} i}\left(x_{\mathrm{D} i}, y_{\mathrm{D} i}, s\right)= & \sum_{n=0}^{\infty} \bar{c}_{i n}(s) \frac{y_{\mathrm{D} i} \sqrt{s} l_{\mathrm{D} i}}{\pi\left\|C_{n}^{(2)}\right\|_{-1}^{2}} \int_{-1}^{1} \frac{\mathrm{K}_{1}\left[\sqrt{s} l_{\mathrm{D} i} \sqrt{\left(x_{\mathrm{D} i}-\xi\right)^{2}+y_{\mathrm{D} i}^{2}}\right]}{\sqrt{\left(x_{\mathrm{D} i}-\xi\right)^{2}+y_{\mathrm{D} i}^{2}}} \\
& \times C_{n}^{(2)}(\xi)\left(1-\xi^{2}\right)^{3 / 2} \mathrm{~d} \xi,
\end{aligned}
$$

where $C_{n}^{(2)}$ denote Gegenbauer polynomials of order $n$ with parameter equal to 2 . Notice that instead of these polynomials any other function can be used, the essential is to keep the multiplier $\left(1-\xi^{2}\right)^{3 / 2}$ to make pressure continuous at the endpoints of the fault. For further information on Gegenbauer polynomials and their properties see, for example, Abramowitz and Stegun (1972). Now let us consider more closely the function

$$
\begin{aligned}
\bar{p}_{\mathrm{D} i n}\left(x_{\mathrm{D} i}, y_{\mathrm{D} i}, s\right)= & \frac{y_{\mathrm{D} i} \sqrt{s} l_{\mathrm{D} i}}{\pi\left\|C_{n}^{(2)}\right\|^{2}} \int_{-1}^{1} \frac{\mathrm{K}_{1}\left[\sqrt{s} l_{\mathrm{D} i} \sqrt{\left(x_{\mathrm{D} i}-\xi\right)^{2}+y_{\mathrm{D} i}^{2}}\right]}{\sqrt{\left(x_{\mathrm{D} i}-\xi\right)^{2}+y_{\mathrm{D} i}^{2}}} \\
& \times C_{n}^{(2)}(\xi)\left(1-\xi^{2}\right)^{3 / 2} \mathrm{~d} \xi .
\end{aligned}
$$

It can be seen that Eq. 61 satisfies Eq. 20 outside the fault. This function itself as well as its derivative are sufficiently easy to evaluate outside the fault (see Appendix for one possible approach to its efficient computation). On the fault, the corresponding integral does not exist and the whole expression should be regarded as a limit as $y_{\mathrm{D} i}$ tends to zero. Using the properties of the Modified Bessel functions given in Abramowitz and Stegun (1972) it can be shown that

$$
\lim _{y_{\mathrm{D} i} \rightarrow \pm 0} \bar{p}_{\mathrm{D} i n}\left(x_{\mathrm{D} i}, y_{\mathrm{D} i}, s\right)= \pm \frac{1}{\pi\left\|C_{n}^{(2)}\right\|^{2}} C_{n}^{(2)}\left(x_{\mathrm{D} i}\right)\left(1-x_{\mathrm{D} i}^{2}\right)^{3 / 2}, \quad-1 \leq x_{\mathrm{D} i} \leq 1 .
$$

The calculation of derivative on the fault is slightly more complicated; however, it can be written in the following form:

$$
\begin{aligned}
\left.\frac{\partial \bar{p}_{\mathrm{D} i n}\left(x_{\mathrm{D} i}, y_{\mathrm{D} i}, s\right)}{\partial y_{\mathrm{D} i}}\right|_{y_{\mathrm{D} i} \rightarrow 0}= & \left.\frac{\partial}{\partial y_{\mathrm{D} i}} \frac{y_{\mathrm{D} i}}{\pi \mid C_{n}^{(2)} \|^{2}} \int_{-1}^{1} \frac{C_{n}^{(2)}(\xi)\left(1-\xi^{2}\right)^{3 / 2}}{\left(x_{\mathrm{D} i}-\xi\right)^{2}+y_{\mathrm{D} i}^{2}} \mathrm{~d} \xi\right|_{y_{\mathrm{D} i} \rightarrow 0}+\frac{\partial}{\partial y_{\mathrm{D} i}} \frac{y_{\mathrm{D} i}}{\pi} \\
& \times \int_{-1}^{1}\left\{\frac{\sqrt{s} l_{\mathrm{D} i} \mathrm{~K}_{1}\left[\sqrt{s} l_{\mathrm{D} i} \sqrt{\left(x_{\mathrm{D} i}-\xi\right)^{2}+y_{\mathrm{D} i}^{2}}\right]}{\sqrt{\left(x_{\mathrm{D} i}-\xi\right)^{2}+y_{\mathrm{D} i}^{2}}}\right. \\
& \left.-\frac{1}{\left(x_{\mathrm{D} i}-\xi\right)^{2}+y_{\mathrm{D} i}^{2}}\right\} \\
& \times\left.\frac{C_{n}^{(2)}(\xi)}{\left\|C_{n}^{(2)}\right\|^{2}}\left(1-\xi^{2}\right)^{3 / 2} \mathrm{~d} \xi\right|_{y_{\mathrm{D} i} \rightarrow 0}
\end{aligned}
$$


It can be seen that the integral in the second term exists for every $y_{\mathrm{D} i}$ (although sub-integral expression has a logarithmic singularity, but it is still integrable), while using the rules for integral differentiation and substitution $\xi=z y_{\mathrm{D} i}+x_{\mathrm{D} i}$, we obtain

$$
\begin{aligned}
\left.\frac{\partial}{\partial y_{\mathrm{D} i}} y_{\mathrm{D} i} \int_{-1}^{1} \frac{C_{n}^{(2)}(\xi)\left(1-\xi^{2}\right)^{3 / 2}}{\left(x_{\mathrm{D} i}-\xi\right)^{2}+y_{\mathrm{D} i}^{2}} \mathrm{~d} \xi\right|_{y_{\mathrm{D} i} \rightarrow 0} \\
=\left.\frac{\partial}{\partial y_{\mathrm{D} i}} \int_{\frac{-1-x_{\mathrm{D} i}}{y_{\mathrm{D} i}}}^{\frac{1-x_{\mathrm{D} i}}{y_{\mathrm{D} i}}} \frac{C_{n}^{(2)}\left(z y_{\mathrm{D} i}+x_{\mathrm{D} i}\right)\left[1-\left(z y_{\mathrm{D} i}+x_{\mathrm{D} i}\right)^{2}\right]^{3 / 2}}{z^{2}+1} \mathrm{~d} z\right|_{y_{\mathrm{D} i} \rightarrow 0} \\
=\left.\int_{-1}^{1} \frac{\left[C_{n}^{(2)}(\xi)\left(1-\xi^{2}\right)^{3 / 2}\right]^{\prime}\left(\xi-x_{\mathrm{D} i}\right)}{\left(x_{\mathrm{D} i}-\xi\right)^{2}+y_{\mathrm{D} i}^{2}} \mathrm{~d} \xi\right|_{y_{\mathrm{D} i} \rightarrow 0} \\
=\left.\int_{-1}^{1} \frac{\left[\omega^{\prime}(\xi)-\omega^{\prime}\left(x_{\mathrm{D} i}\right)\right]\left(\xi-x_{\mathrm{D} i}\right)}{\left(x_{\mathrm{D} i}-\xi\right)^{2}+y_{\mathrm{D} i}^{2}} \mathrm{~d} \xi\right|_{y_{\mathrm{D} i} \rightarrow 0} \\
\quad-\left.\omega^{\prime}\left(x_{\mathrm{D} i}\right) \int_{-1}^{1} \frac{\left(\xi-x_{\mathrm{D} i}\right)}{\left(x_{\mathrm{D} i}-\xi\right)^{2}+y_{\mathrm{D} i}^{2}} \mathrm{~d}\right|_{y_{\mathrm{D} i} \rightarrow 0} \\
=\int_{-1}^{1} \frac{\left[\omega^{\prime}(\xi)-\omega^{\prime}\left(x_{\mathrm{D} i}\right)\right]}{\left(\xi-x_{\mathrm{D} i}\right)} \mathrm{d} \xi-\omega^{\prime}\left(x_{\mathrm{D} i}\right) \ln \left|\frac{1-x_{\mathrm{D} i}}{1+x_{\mathrm{D} i}}\right|
\end{aligned}
$$

where $\omega(\xi)=C_{n}^{(2)}(\xi)\left(1-\xi^{2}\right)^{3 / 2}$. The last expression exists due to smoothness of its derivative in the interval $(-1,1)$ and the fact that it vanishes at -1 and 1 . Finally, we obtain

$$
\begin{aligned}
& \left.\frac{\partial \bar{p}_{\mathrm{D} i n}\left(x_{\mathrm{D} i}, y_{\mathrm{D} i}, s\right)}{\partial y_{\mathrm{D} i}}\right|_{y_{\mathrm{D} i} \rightarrow 0}=\frac{1}{\pi\left\|C_{n}^{(2)}\right\|^{2}}\left\{\int_{-1}^{1} \frac{\left[\omega^{\prime}(\xi)-\omega^{\prime}\left(x_{\mathrm{D} i}\right)\right]}{\left(\xi-x_{\mathrm{D} i}\right)} \mathrm{d} \xi-\omega^{\prime}\left(x_{\mathrm{D} i}\right) \ln \left|\frac{1-x_{\mathrm{D} i}}{1+x_{\mathrm{D} i}}\right|\right. \\
& \left.+\int_{-1}^{1}\left[\frac{\sqrt{s} l_{\mathrm{D} i} \mathrm{~K}_{1}\left(\sqrt{s} l_{\mathrm{D} i}\left|x_{\mathrm{D} i}-\xi\right|\right)}{\left|x_{\mathrm{D} i}-\xi\right|}-\frac{1}{\left(x_{\mathrm{D} i}-\xi\right)^{2}}\right] C_{n}^{(2)}(\xi)\left(1-\xi^{2}\right)^{3 / 2} \mathrm{~d} \xi\right\} .
\end{aligned}
$$

Now, the newly defined function $\bar{p}_{\mathrm{D} i}$ together with its derivative can be sufficiently easy evaluated everywhere in reservoir, and thus we can use it in Eqs. 32-35 together with the boundary condition Eq. 58 to account for presence of the non- or low-conductive faults in the reservoir. As collocation points on faults we suggest to take Gegenbauer-Gauss quadrature nodes and to evaluate the integrals using Gegenbauer-Gauss quadrature rule (see Appendix) because it corresponds to expansion of the "generating" functions on the fault in terms of Gegenbauer polynomials in the sense of quadrature rules. Note that due to the continuity of all other functions on the $i$ th fault, only $\bar{p}_{\mathrm{D} i}$ will contribute to the pressure difference term in Eq. 58, so that it can be rewritten as 


$$
\begin{aligned}
& \sum_{k=1}^{M} \sum_{n} \bar{c}_{k n} \frac{\partial \bar{p}_{\mathrm{D} k n}}{\partial y_{\mathrm{D} i}}\left[x_{\mathrm{D} i}\left(x_{\mathrm{D} k}, y_{\mathrm{D} k}\right), y_{\mathrm{D} i}\left(x_{\mathrm{D} k}, y_{\mathrm{D} k}\right), s\right] \\
& \quad=\frac{2 \alpha_{\mathrm{D} i}}{\pi} \sum_{n} \bar{c}_{i n} \frac{C_{n}^{(2)}\left(x_{\mathrm{D} i}\right)\left(1-x_{\mathrm{D} i}^{2}\right)^{3 / 2}}{\left\|C_{n}^{(2)}\right\|^{2}}, \quad-1 \leq x_{\mathrm{D} i} \leq 1 .
\end{aligned}
$$

\subsection{Composite Systems and Reservoirs with Boundaries}

Finite reservoir boundaries and composite limits can be modeled using a standard boundary elements technique [see, for example, Brebbia (1978)]. For the sake of completeness, we present a slightly different approach which is based on using continuous functions rather than discontinuous elements. Let us assume that the boundary (or a composite limit) is a closed curve $\gamma$ defined in its coordinate system (the orientation of the coordinate system is not important here, but the origin should be "inside" the curve) as follows:

$$
\begin{cases}x_{\mathrm{D} \gamma}(\varphi)=u_{\gamma}(\varphi), & 0 \leq \varphi \leq 2 \pi, \\ y_{\mathrm{D} \gamma}(\varphi)=v_{\gamma}(\varphi), & 0 \leq \varphi \leq 2 \pi,\end{cases}
$$

and that the source intensity distribution on this curve is given as

$$
\frac{f_{\gamma}(\varphi, s)}{\sqrt{\left[u_{\gamma}^{\prime}(\varphi)\right]^{2}+\left[v_{\gamma}^{\prime}(\varphi)^{2}\right]}}
$$

where ' denotes the derivative. In this case, the pressure change in the reservoir induced by such source distribution can be written as follows:

$$
\bar{p}_{\mathrm{D} \gamma}\left(x_{\mathrm{D} \gamma}, y_{\mathrm{D}_{\gamma}}, s\right)=\frac{1}{\pi} \int_{0}^{2 \pi} \mathrm{K}_{0}\left\{\sqrt{s} \sqrt{\left[x_{\mathrm{D} \gamma}-u_{\gamma}(\varphi)\right]^{2}+\left[y_{\mathrm{D} \gamma}-v_{\gamma}(\varphi)\right]^{2}}\right\} f_{\gamma}(\varphi, s) \mathrm{d} \varphi .
$$

To evaluate this function at any point of the reservoir one can use the same techniques as for the fracture case. However, it might be necessary to evaluate the outer normal derivative exactly on the boundary (for example to honor the no-flow condition) which can be complicated. Using the properties of the Modified Bessel functions it can be shown that

$$
\begin{aligned}
& \frac{\partial \bar{p}_{\mathrm{D} \gamma}}{\partial n_{ \pm}}\left(v_{\gamma}(\psi), u_{\gamma}(\psi), s\right) \\
& =f_{\gamma}(\psi) \pm \frac{\sqrt{s}}{2 \pi} \int_{0}^{2 \pi} \mathrm{K}_{1}\left\{\sqrt{s} \sqrt{\left[u_{\gamma}(\psi)-u_{\gamma}(\varphi)\right]^{2}+\left[v_{\gamma}(\psi)-v_{\gamma}(\varphi)\right]^{2}}\right\} \\
& \quad \times \frac{\left[u_{\gamma}(\varphi)-u_{\gamma}(\psi)\right] v_{\gamma}^{\prime}(\psi)-\left[v_{\gamma}(\varphi)-v_{\gamma}(\psi)\right] u_{\gamma}^{\prime}(\psi)}{\sqrt{\left[u_{\gamma}(\varphi)-u_{\gamma}(\psi)\right]^{2}+\left[v_{\gamma}(\varphi)-v_{\gamma}(\psi)\right]^{2}}} f_{\gamma}(\varphi, s) \mathrm{d} \varphi, \quad 0 \leq \psi \leq 2 \pi .
\end{aligned}
$$

The "+" sign corresponds to the inner normal to the boundary defined by Eq. 67, and "-" to the outer normal to the same boundary. Using Taylor's decomposition, it is easy to see that sub-integral expression is non-singular as long as $u_{\gamma}$ and $v_{\gamma}$ are twice differentiable and 
$\sqrt{\left(u_{\gamma}^{\prime}\right)^{2}+\left(v_{\gamma}^{\prime}\right)^{2}}$ is positive everywhere on the curve (condition of non-degeneration). Now, if we assume that $f_{\gamma}$ can be expanded into a series as

$$
f_{\gamma}(\varphi, s)=\sum_{n} \bar{c}_{\gamma n}(s) f_{\gamma n}(\varphi)
$$

we can define the function representing the pressure change induced by the boundary $\gamma$ as follows:

$$
\bar{p}_{\mathrm{D} \gamma}\left(x_{\mathrm{D} \gamma}, y_{\mathrm{D}_{\gamma}}, s\right)=\sum_{n} \bar{c}_{\gamma n} \bar{p}_{\mathrm{D} \gamma n}\left(x_{\mathrm{D} \gamma}, y_{\mathrm{D}_{\gamma}}, s\right),
$$

where

$$
\bar{p}_{\mathrm{D} \gamma n}\left(x_{\mathrm{D} \gamma}, y_{\mathrm{D}_{\gamma}}, s\right)=\frac{1}{\pi} \int_{0}^{2 \pi} \mathrm{K}_{0}\left\{\sqrt{s} \sqrt{\left[x_{\mathrm{D} \gamma}-u_{\gamma}(\varphi)\right]^{2}+\left[y_{\mathrm{D} \gamma}-v_{\gamma}(\varphi)\right]^{2}}\right\} f_{\gamma n}(\varphi) \mathrm{d} \varphi .
$$

Note that as $\left\{f_{\gamma_{n}}\right\}_{n}$ we can take any complete system of orthogonal functions like $\cos n \varphi, \sin m \varphi$, or any other. Depending on the nature of the process, the condition on boundary might be one of the following:

- Constant pressure boundary

$$
\sum_{k=1}^{M} \sum_{n} \bar{c}_{k n} \bar{p}_{\mathrm{D} k n}\left[x_{\mathrm{D}}\left(x_{\mathrm{D} k}, y_{\mathrm{D} k}\right), y_{\mathrm{D}}\left(x_{\mathrm{D} k}, y_{\mathrm{D} k}\right), s\right]=0 \text { on } \gamma,
$$

where $n$ runs through the indexes of features lying only inside or outside of the boundary.

- No-flow boundary

$$
\sum_{k=1}^{M} \sum_{n} \bar{c}_{k n} \frac{\partial \bar{p}_{\mathrm{D} k n}}{\partial n_{ \pm}}\left[x_{\mathrm{D}}\left(x_{\mathrm{D} k}, y_{\mathrm{D} k}\right), y_{\mathrm{D}}\left(x_{\mathrm{D} k}, y_{\mathrm{D} k}\right), s\right]=0 \quad \text { on } \gamma .
$$

The "+" sign corresponds the normal coming inwards, and thus to outer boundary ( $n$ runs only through indexes of features that are inside the boundary) and "-" to the one coming outwards, and thus to the inner boundary ( $n$ runs only through indexes of features that are outside of the boundary).

- Composite limit boundary If the boundary separates two regions - the inner with the values of fluid and rock properties equal to $\phi_{+}, c_{\mathrm{t}+}, k_{+}, \mu_{+}$and the outer with $\phi_{-}, c_{\mathrm{t}-}, k_{-}, \mu_{-}$, then, assuming that all dimensionless variables are defined with respect to some reference values of $\phi, c_{\mathrm{t}}, k$, and $\mu$, the following conditions must be satisfied on the boundary:

$$
\begin{aligned}
& \sum_{k=1}^{M} \sum_{n \in \text { inside }} \bar{c}_{k n} \bar{p}_{\mathrm{D} k n}\left[x_{\mathrm{D}}\left(x_{\mathrm{D} k}, y_{\mathrm{D} k}\right), y_{\mathrm{D}}\left(x_{\mathrm{D} k}, y_{\mathrm{D} k}\right), s \frac{\phi_{+} \mu_{+} c_{\mathrm{t}+} k}{\phi \mu c_{\mathrm{t}} k_{+}}\right] \\
& =\sum_{k=1}^{M} \sum_{n \in \text { outside }} \bar{c}_{k n} \bar{p}_{\mathrm{D} k n}\left[x_{\mathrm{D}}\left(x_{\mathrm{D} k}, y_{\mathrm{D} k}\right), y_{\mathrm{D}}\left(x_{\mathrm{D} k}, y_{\mathrm{D} k}\right), s \frac{\phi_{-} \mu_{-} c_{\mathrm{t}-}}{\phi \mu c_{\mathrm{t}} k_{-}}\right] \text {on } \gamma,
\end{aligned}
$$




$$
\begin{aligned}
& -\frac{k_{+} \mu}{k \mu_{+}} \sum_{k=1}^{M} \sum_{n \in \text { inside }} \bar{c}_{k n} \frac{\partial \bar{p}_{\mathrm{D} k n}}{\partial n_{+}}\left[x_{\mathrm{D}}\left(x_{\mathrm{D} k}, y_{\mathrm{D} k}\right), y_{\mathrm{D}}\left(x_{\mathrm{D} k}, y_{\mathrm{D} k}\right), s \frac{\phi_{+} \mu_{+} c_{\mathrm{t}+} k}{\phi \mu c_{\mathrm{t}} k_{+}}\right] \\
& =\frac{k_{-} \mu}{k \mu_{-}} \sum_{k=1}^{M} \sum_{n \in \text { outside }} \bar{c}_{k n} \frac{\partial \bar{p}_{\mathrm{D} k n}}{\partial n_{-}}\left[x_{\mathrm{D}}\left(x_{\mathrm{D} k}, y_{\mathrm{D} k}\right), y_{\mathrm{D}}\left(x_{\mathrm{D} k}, y_{\mathrm{D} k}\right), s \frac{\phi_{-} \mu_{-} c_{\mathrm{t}-k}}{\phi \mu c_{\mathrm{t}} k_{-}}\right] \text {on } \gamma .
\end{aligned}
$$

Other boundary conditions are also possible and they may be written in the same way.

\subsection{Transient Solution in Time Domain}

The solutions presented above can be simplified in a certain sense if we rewrite them in time domain. Really, applying the inverse Laplace transform to Eqs. 32-35 we can obtain the following system:

$$
\begin{gathered}
\sum_{n=0}^{\infty} a_{n} \otimes p_{\mathrm{D} 0 n 1}\left[x_{\mathrm{D}}\left(r_{\mathrm{D}}, \varphi\right), y_{\mathrm{D}}\left(r_{\mathrm{D}}, \varphi\right)\right]+\sum_{n=1}^{\infty} b_{n} \otimes p_{\mathrm{D} 0 n 2}\left[x_{\mathrm{D}}\left(r_{\mathrm{D}}, \varphi\right), y_{\mathrm{D}}\left(r_{\mathrm{D}}, \varphi\right)\right] \\
+\sum_{k=1}^{M} \sum_{n=0}^{\infty} c_{k n} \otimes p_{\mathrm{D} k n}\left[x_{\mathrm{D}}\left(x_{\mathrm{D} k}, y_{\mathrm{D} k}\right), y_{\mathrm{D}}\left(x_{\mathrm{D} k}, y_{\mathrm{D} k}\right)\right]=p_{\mathrm{Dw}}\left(t_{\mathrm{D}}\right) \text { on } \Gamma_{i}, i \in I_{0}, \\
a_{0}+\sum_{i \in I_{0}} c_{i 0}=q_{\mathrm{D}}\left(t_{\mathrm{D}}\right), \\
\sum_{n=0}^{\infty} a_{n} \otimes p_{\mathrm{D} 0 n 1}\left[x_{\mathrm{D}}\left(r_{\mathrm{D}}, \varphi\right), y_{\mathrm{D}}\left(r_{\mathrm{D}}, \varphi\right)\right]+\sum_{n=1}^{\infty} b_{n} \otimes p_{\mathrm{D} 0 n 2}\left[x_{\mathrm{D}}\left(r_{\mathrm{D}}, \varphi\right), y_{\mathrm{D}}\left(r_{\mathrm{D}}, \varphi\right)\right] \\
+\sum_{k=1}^{M} \sum_{n=0}^{\infty} c_{k n} \otimes p_{\mathrm{D} k n}\left[x_{\mathrm{D}}\left(x_{\mathrm{D} k}, y_{\mathrm{D} k}\right), y_{\mathrm{D}}\left(x_{\mathrm{D} k}, y_{\mathrm{D} k}\right)\right]=p_{\mathrm{Df} j}\left(t_{\mathrm{D}}\right) \text { on } \Gamma_{i}, \quad i \in I_{j}, j>0, \\
\sum_{i \in I_{j}} c_{i 0}=0, \quad j>0,
\end{gathered}
$$

where " $\otimes$ " symbol denotes convolution operation, i.e., $f(t) \otimes h(t)=\int_{0}^{t} f(t-\tau) h(\tau) \mathrm{d} \tau$. Now, choosing a certain uniform time grid $t_{\mathrm{D} g}=g \Delta t_{\mathrm{D}}$ and assuming that unknown functions $a_{n}, b_{n}, c_{k n}$ are constant within each interval $\left[(g-1) \Delta t_{\mathrm{D}}, g \Delta t_{\mathrm{D}}\right]$ and taking on it values of $a_{n}^{g}, b_{n}^{g}$ and $c_{k n}^{g}$

$$
\begin{aligned}
& \sum_{n=0}^{\infty} \sum_{g=0}^{G} a_{n}^{g} P_{\mathrm{D} 0 n 1}^{G-g}\left[x_{\mathrm{D}}\left(r_{\mathrm{D}}, \varphi\right), y_{\mathrm{D}}\left(r_{\mathrm{D}}, \varphi\right)\right]+\sum_{n=1}^{\infty} \sum_{g=0}^{G} b_{n}^{g} P_{\mathrm{D} 0 n 2}^{G-g}\left(x_{\mathrm{D}}\left(r_{\mathrm{D}}, \varphi\right), y_{\mathrm{D}}\left(r_{\mathrm{D}}, \varphi\right)\right) \\
& \quad+\sum_{k=1}^{M} \sum_{n=0}^{\infty} \sum_{g=0}^{G} c_{k n}^{g} P_{\mathrm{D} k n}^{G-g}\left[x_{\mathrm{D}}\left(x_{\mathrm{D} k}, y_{\mathrm{D} k}\right), y_{\mathrm{D}}\left(x_{\mathrm{D} k}, y_{\mathrm{D} k}\right)\right]=p_{\mathrm{Dw}}\left(t_{\mathrm{D} G}\right) \quad \text { on } \Gamma_{i}, i \in I_{0}, \\
& a_{0}^{G}+\sum_{i \in I_{0}} c_{i 0}^{G}=q_{\mathrm{D}}\left(t_{\mathrm{D} G}\right),
\end{aligned}
$$




$$
\begin{aligned}
& \sum_{n=0}^{\infty} \sum_{g=0}^{G} a_{n}^{g} P_{\mathrm{D} 0 n 1}^{G-g}\left[x_{\mathrm{D}}\left(r_{\mathrm{D}}, \varphi\right), y_{\mathrm{D}}\left(r_{\mathrm{D}}, \varphi\right)\right]+\sum_{n=1}^{\infty} \sum_{g=0}^{G} b_{n}^{r} P_{\mathrm{D} 0 n 2}^{G-g}\left(x_{\mathrm{D}}\left(r_{\mathrm{D}}, \varphi\right), y_{\mathrm{D}}\left(r_{\mathrm{D}}, \varphi\right)\right) \\
& +\sum_{k=1}^{M} \sum_{n=0}^{\infty} \sum_{g=0}^{G} c_{k n}^{g} P_{\mathrm{D} k n}^{G-g}\left[x_{\mathrm{D}}\left(x_{\mathrm{D} k}, y_{\mathrm{D} k}\right), y_{\mathrm{D}}\left(x_{\mathrm{D} k}, y_{\mathrm{D} k}\right)\right]=p_{\mathrm{Df} j}\left(t_{\mathrm{D} G}\right) \text { on } \Gamma_{i}, i \in I_{j}, j>0, \\
& \sum_{i \in I_{j}} c_{i 0}^{G}=0, \quad j>0,
\end{aligned}
$$

where

$$
\begin{aligned}
& P_{\mathrm{D} 0 n 1}^{g}\left[x_{\mathrm{D}}\left(r_{\mathrm{D}}, \varphi\right), y_{\mathrm{D}}\left(r_{\mathrm{D}}, \varphi\right)\right]=\int_{t_{\mathrm{D}(g-1)}}^{t_{\mathrm{D} g}} L^{-1}\left\{\frac{2 \mathrm{~K}_{n}\left(\sqrt{s} r_{\mathrm{D}}\right) \cos n \varphi}{\left[\mathrm{K}_{n+1}\left(\sqrt{s} r_{\mathrm{Dw}}\right)+\mathrm{K}_{n-1}\left(\sqrt{s} r_{\mathrm{Dw}}\right)\right] \sqrt{s} r_{\mathrm{Dw}}}\right\} \mathrm{d} \tau, \\
& P_{\mathrm{D} 0 n 2}^{g}\left[x_{\mathrm{D}}\left(r_{\mathrm{D}}, \varphi\right), y_{\mathrm{D}}\left(r_{\mathrm{D}}, \varphi\right)\right]=\int_{t_{\mathrm{D}(g-1)}}^{-1}\left\{\frac{2 \mathrm{~K}_{n}\left(\sqrt{s} r_{\mathrm{D}}\right) \sin n \varphi}{\left[\mathrm{K}_{n+1}\left(\sqrt{s} r_{\mathrm{Dw}}\right)+\mathrm{K}_{n-1}\left(\sqrt{s} r_{\mathrm{Dw}}\right)\right] \sqrt{s} r_{\mathrm{Dw}}}\right\} \mathrm{d} \tau, \\
& P_{\mathrm{D} i n}^{g}\left[x_{\mathrm{D}}\left(x_{\mathrm{D} i}, y_{\mathrm{D} i}\right), y_{\mathrm{D}}\left(x_{\mathrm{D} i}, y_{\mathrm{D} i}\right)\right]=\int_{t_{\mathrm{D}(g-1)}}^{t_{\mathrm{D} g}} \frac{1}{2 \tau \pi}\left\{\int_{0}^{\pi} e^{-\frac{l_{\mathrm{D} i}^{2}\left[\left(x_{\mathrm{D} i}-\cos \psi\right)^{2}+y_{\mathrm{D} i}^{2}\right]}{4 \tau}} \cos n \psi \mathrm{d} \psi\right\} \mathrm{d} \tau,
\end{aligned}
$$

and $L^{-1}$ denotes the inverse Laplace transform.

Carrying out integration in time domain, we can rewrite these functions as follows:

$$
\begin{aligned}
P_{\mathrm{D} 0 n 1}^{g}\left(x_{\mathrm{D}}\left(r_{\mathrm{D}}, \varphi\right), y_{\mathrm{D}}\left(r_{\mathrm{D}}, \varphi\right)\right) & =\left.\mathrm{W}_{n}\left(\frac{\tau}{r_{\mathrm{Dw}}^{2}}, \frac{r_{\mathrm{D}}}{r_{\mathrm{Dw}}}\right)\right|_{t_{\mathrm{D}(g-1)}} ^{t_{\mathrm{D} g}} \cos \varphi, \\
P_{\mathrm{D} 0 n 2}^{g}\left(x_{\mathrm{D}}\left(r_{\mathrm{D}}, \varphi\right), y_{\mathrm{D}}\left(r_{\mathrm{D}}, \varphi\right)\right) & =\left.\mathrm{W}_{n}\left(\frac{\tau}{r_{\mathrm{Dw}}^{2}}, \frac{r_{\mathrm{D}}}{r_{\mathrm{Dw}}}\right)\right|_{t_{\mathrm{D}(g-1)}} ^{t_{\mathrm{D} g}} \sin \varphi, \\
P_{\mathrm{D} i n}^{g}\left(x_{\mathrm{D}}\left(x_{\mathrm{D} i}, y_{\mathrm{D} i}\right), y_{\mathrm{D}}\left(x_{\mathrm{D} i}, y_{\mathrm{D} i}\right)\right) & =\left.\frac{1}{2 \pi} \int_{0}^{\pi} \mathrm{E}_{1}\left[\frac{\left(x_{\mathrm{D} i}-\cos \psi\right)^{2}+y_{\mathrm{D} i}^{2}}{4 \tau / l_{\mathrm{D} i}^{2}}\right]\right|_{t_{\mathrm{D}(g-1)}} ^{t_{\mathrm{D} g}} \cos n \psi \mathrm{d} \psi,
\end{aligned}
$$

where $\mathrm{E}_{1}$ denotes exponential integral and

$$
\mathrm{W}_{n}\left(\tau, r_{\mathrm{D}}\right)=L^{-1}\left\{\frac{2 \mathrm{~K}_{n}\left(\sqrt{s} r_{\mathrm{D}}\right)}{\left[\mathrm{K}_{n+1}(\sqrt{s})+\mathrm{K}_{n-1}(\sqrt{s})\right] s \sqrt{s}}\right\} .
$$

The system defined by Eqs. 81-84 is solved by a stepping procedure starting from initial moment of time which is to be increased on every new step. Due to the presence of convolution operation it is only convenient to solve the system with constant time step. However, computations show that this scheme is very robust and its accuracy is almost independent of time step. It gives the values very close to the ones obtained from solution in Laplace domain for a very wide range of time steps (at least for $10^{-4}<\Delta t_{\mathrm{D}}<10^{4}$ ), provided 
that the total flow rate $q_{\mathrm{D}}\left(t_{\mathrm{D}}\right)$ is independent of time. The last fact should not be considered as a restriction since it can be easily overcome by using convolution integral to obtain the solution for a time-dependent flow rate. Finally, let us note that this approach proves to be much faster than the one for Laplace domain. The same technique without any significant changes can be also applied to finite-conductivity fractures case to convert the corresponding solution from Laplace to time domain. The modeling of faults requires special consideration since in this case the functions $\bar{p}_{\mathrm{D} k n}$ are expressed in terms of Modified Bessel functions of the first order (see Eqs. 61, 65). In time domain, we suggest to take the following function:

$$
\begin{aligned}
& P_{\mathrm{D} i n}^{g}\left[x_{\mathrm{D}}\left(x_{\mathrm{D} i}, y_{\mathrm{D} i}\right), y_{\mathrm{D}}\left(x_{\mathrm{D} i}, y_{\mathrm{D} i}\right)\right] \\
& =\frac{y_{\mathrm{D} i}}{\pi} \int_{t_{\mathrm{D}(g-1)}}^{t_{\mathrm{D} g}} \int_{-1}^{1} L^{-1}\left\{\frac{\mathrm{K}_{1}\left[\sqrt{s} l_{\mathrm{D} i} \sqrt{\left(x_{\mathrm{D} i}-\xi\right)^{2}+y_{\mathrm{D} i}^{2}}\right]}{\sqrt{s} l_{\mathrm{D} i} \sqrt{\left(x_{\mathrm{D} i}-\xi\right)^{2}+y_{\mathrm{D} i}^{2}}}\right\} \frac{C_{n}^{2}(\xi)}{\left\|C_{n}^{2}\right\|^{2}}\left(1-\xi^{2}\right)^{3 / 2} \mathrm{~d} \xi \mathrm{d} \tau \\
& =\frac{y_{\mathrm{D} i}}{\pi} \int_{-1}^{1}\left\{\frac{\mathrm{E}_{2}\left[\frac{\left(x_{\mathrm{D} i}-\xi\right)^{2}+y_{\mathrm{D} i}^{2}}{4 t_{\mathrm{D} g} / l_{\mathrm{D} i}^{2}}\right]}{\left[\frac{\left(x_{\mathrm{D} i}-\xi\right)^{2}+y_{\mathrm{D} i}^{2}}{t_{\mathrm{D} g} / l_{\mathrm{D} i}^{2}}\right]}-\frac{\mathrm{E}_{2}\left[\frac{\left(x_{\mathrm{D} i}-\xi\right)^{2}+y_{\mathrm{D} i}^{2}}{4 t_{\mathrm{D}(g-1)} / l_{\mathrm{D} i}^{2}}\right]}{\left[\frac{\left(x_{\mathrm{D} i}-\xi\right)^{2}+y_{\mathrm{D} i}^{2}}{t_{\mathrm{D}(g-1)} / l_{\mathrm{D} i}^{2}}\right]}\right\} \frac{C_{n}^{2}(\xi)}{\left\|C_{n}^{2}\right\|^{2}}\left(1-\xi^{2}\right)^{3 / 2} \mathrm{~d} \xi
\end{aligned}
$$

where $\mathrm{E}_{2}(x)=e^{-x}-x \mathrm{E}_{1}(x)$. Note that it corresponds to the one defined by Eq. 61 divided by $s l_{\mathrm{D} i}$, since it provides better accuracy in time domain than the original one. Eq. 92 also allows to find the corresponding expression for the normal derivative on the fault from Eq. 65 .

Similarly, to account for boundaries in time domain we suggest to use the following function:

$$
\begin{aligned}
& P_{\mathrm{D} \gamma n}^{g}\left[x_{\mathrm{D}}\left(x_{\mathrm{D} \gamma}, y_{\mathrm{D} \gamma}\right), y_{\mathrm{D}}\left(x_{\mathrm{D} \gamma}, y_{\mathrm{D} \gamma}\right)\right] \\
& =\frac{1}{\pi} \int_{t_{\mathrm{D}(r-1)}}^{t_{\mathrm{D} g}} \int_{0}^{2 \pi} L^{-1}\left(\frac{\mathrm{K}_{0}\left\{\sqrt{s} \sqrt{\left[x_{\mathrm{D} \gamma}-u_{\gamma}(\varphi)\right]^{2}+\left[y_{\mathrm{D} \gamma}-v_{\gamma}(\varphi)\right]^{2}}\right\}}{s} f_{\gamma n}(\varphi)\right) \mathrm{d} \varphi \mathrm{d} \tau \\
& =\left.\frac{1}{2 \pi} \int_{0}^{2 \pi} \tau \mathrm{U}\left\{\frac{\sqrt{\left[x_{\mathrm{D} \gamma}-u_{\gamma}(\varphi)\right]^{2}+\left[y_{\mathrm{D} \gamma}-v_{\gamma}(\varphi)\right]^{2}}}{\tau}\right\}\right|_{t_{\mathrm{D}(g-1)}} ^{t_{\mathrm{D} g}} f_{\gamma n}(\varphi) \mathrm{d} \varphi
\end{aligned}
$$

where $\mathrm{U}(x)=\mathrm{E}_{1}(x)-\mathrm{E}_{2}(x)$. Again this function corresponds to the one defined by Eq. 69 multiplied by $1 / s$ to achieve better accuracy in time domain. Its normal derivative on the boundary can be easily obtained from Eq. 70 by using Eq. 92 .

\section{Examples}

The solutions given in the previous sections are compared in this section with some wellknown analytical solutions given in the literature. First, we compare the uniform pressure solution given in Sect. 2.1 and 2.2 with the solution given by Riley et al. (2007). They obtained the dimensionless pressure for a vertical fracture with finite conductivity, where the fracture is assumed to have an elliptical cross section, the flow within the fracture to be incompressible, and the reservoir to be infinite. It is a line-source solution, from which they computed the 
Table 1 Comparison of dimensionless pressures from Riley et al. (2007) and this article

\begin{tabular}{|c|c|c|c|c|c|c|c|c|}
\hline \multirow[t]{2}{*}{$t_{\mathrm{D}}$} & \multicolumn{2}{|l|}{$F_{\mathrm{D}}=1$} & \multicolumn{2}{|c|}{$F_{\mathrm{D}}=10$} & \multicolumn{2}{|c|}{$F_{\mathrm{D}}=100$} & \multicolumn{2}{|c|}{$F_{\mathrm{D}}=1,000$} \\
\hline & $p_{\mathrm{D}}^{\mathrm{a}}$ & $p_{\mathrm{D}}^{\mathrm{b}}$ & $p_{\mathrm{D}}^{\mathrm{a}}$ & $p_{\mathrm{D}}^{\mathrm{b}}$ & $p_{\mathrm{D}}^{\mathrm{a}}$ & $p_{\mathrm{D}}^{\mathrm{b}}$ & $p_{\mathrm{D}}^{\mathrm{a}}$ & $p_{\mathrm{D}}^{\mathrm{b}}$ \\
\hline 0.0001 & 0.2406 & 0.2441 & 0.077 & 0.07797 & 0.027 & 0.0279 & 0.0183 & 0.01876 \\
\hline 0.001 & 0.4269 & 0.4311 & 0.1384 & 0.1411 & 0.0656 & 0.6635 & 0.0563 & 0.05641 \\
\hline 0.01 & 0.7447 & 0.7527 & 0.2696 & 0.2756 & 0.1806 & 0.1815 & 0.1708 & 0.1709 \\
\hline 0.1 & 1.2661 & 1.291 & 0.6048 & 0.6148 & 0.5025 & 0.5037 & 0.4916 & 0.4917 \\
\hline 1 & 2.1125 & 2.157 & 1.3386 & 1.353 & 1.2218 & 1.224 & 1.2095 & 1.210 \\
\hline 10 & 3.2042 & 3.255 & 2.3977 & 2.414 & 2.2757 & 2.278 & 2.2628 & 2.263 \\
\hline 100 & 4.3491 & 4.401 & 3.5386 & 3.555 & 3.4158 & 3.418 & 3.4029 & 3.403 \\
\hline 1000 & 5.4998 & 5.552 & 4.6888 & 4.705 & 4.566 & 4.568 & 4.553 & 4.553 \\
\hline
\end{tabular}

${ }^{\mathrm{a}}$ From this article

${ }^{\mathrm{b}}$ From Riley et al. (2007)

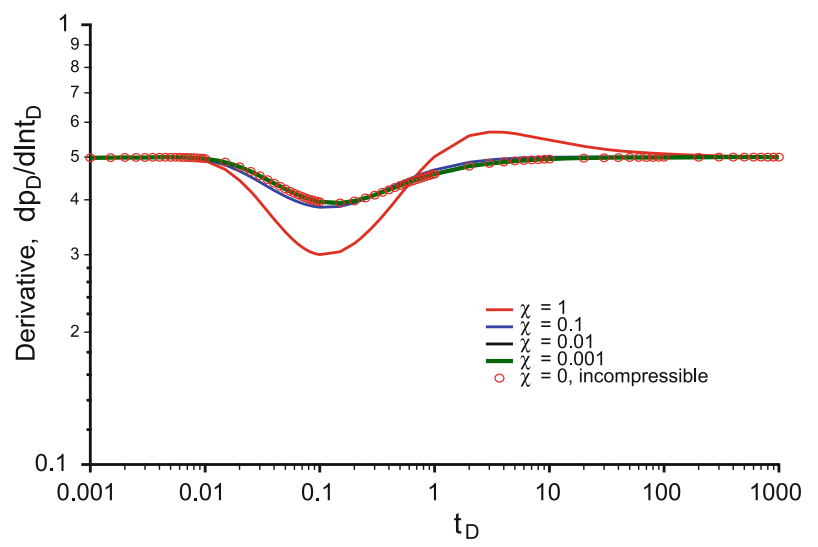

Fig. 10 Comparison of pressure derivatives for different matrix-fracture diffusivity ratios $\left(\chi_{\mathrm{f} i}\right)$ with the derivative of the incompressible fracture solution $\left(\chi_{\mathrm{f} i}=0\right)$

wellbore pressure by using an equivalent wellbore radius. This is a reasonable approximation for the uniform wellbore pressure condition if the time is not very small. Table 1 compares the dimensionless pressures for various fracture conductivities from this article, and from Table 3 of the Riley et al. (2007) article. All dimensionless pressures given in Table 1 are computed at the wellbore. As can be seen, our results are lower than theirs by about $1 \%$, which might be because they used an equivalent wellbore radius with a line-source solution, whereas we used a finite (actual) wellbore radius (in the dimensionless form $r_{\text {Dw }}=10^{-3}$ ) with the uniform pressure boundary condition.

In the previous section, we also presented the solutions for compressible fluid flow inside finite-conductivity fractures to investigate whether the incompressible fracture fluid-flow solutions are accurate enough to be used for naturally fractured reservoirs, where in reality the fluid inside the fractures is compressible. The comparison of the solutions for different matrix-fracture diffusivity ratios $\left(\chi_{\mathrm{f} i}\right.$, given by Eq. 55$)$ with the solution for the incompressible fracture flow $\left(\chi_{\mathrm{f} i}=0\right)$ for $F_{\mathrm{D} i}=1$, is shown in Fig. 10. As can be seen from this figure, there is no discernible difference between the incompressible and compressible solutions for the $\chi_{\mathrm{f} i}$ values of 0.001 and 0.01 , and very little difference for 0.1 . These $\chi_{\mathrm{f} i}$ values cover 


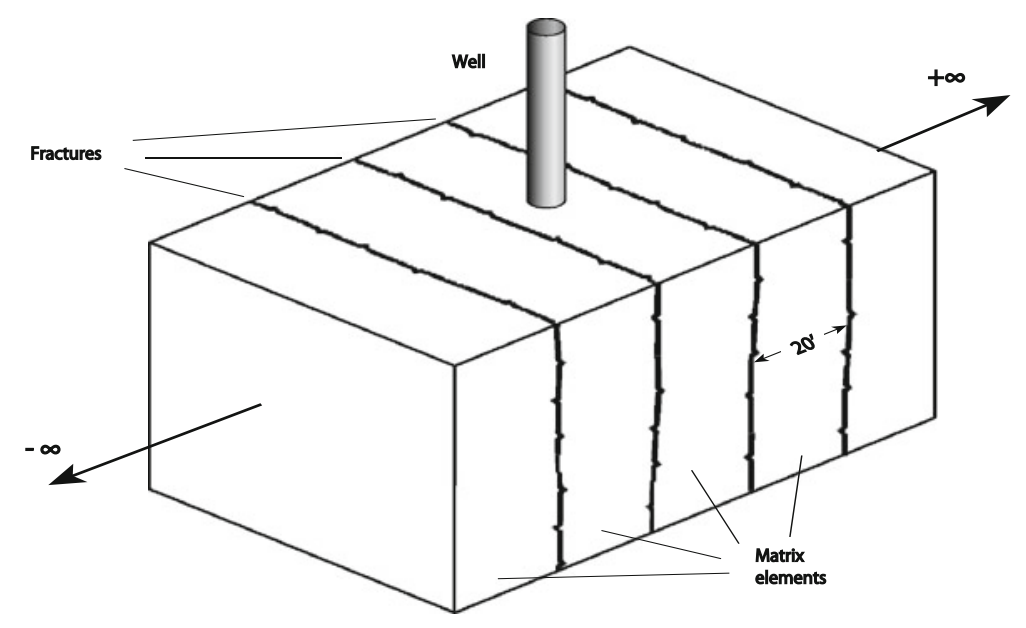

Fig. 11 A schematic of a periodically and discretely fractured reservoir

all possible matrix-fracture properties. There is a noticeable difference between the incompressible and compressible solutions for $\chi_{\mathrm{f} i}=1$. However, the $\chi_{\mathrm{f} i}$ values greater than 0.1 (which are approximately the ratio of the matrix permeability to the fracture permeability) normally do not occur in naturally fractured reservoirs.

We also compare our solution with the one given by Kuchuk and Habashy (1997) for a periodically discretely fractured reservoir, shown in Fig. 11, where fractures are parallel to each other with an infinite length in the $y$ direction. This solution assumes compressible fluid flow inside finite-conductivity fractures. This reservoir contains 40 parallel equally spaced finite-conductivity fractures; the distance between two adjacent fractures is $20 \mathrm{ft}$. For convenience we used $l=200 \mathrm{ft}$ (i.e., 10 times the distance between two neighboring fractures) as a characteristic length with the resulting dimensionless conductivity $F_{\mathrm{D}}=5$ from Eq. 38 . The well is located in the formation between the 20th and 21st fractures (Fig. 11), 5 and $15 \mathrm{ft}$, respectively, away from them. All fractures in the model have the same conductivity.

We used the same model to obtain the pressure behavior of the system, but considered finite-length fractures, with $l_{\mathrm{D} i}=10$; i.e., all fractures are equal in length, which is 200 times greater than the distance between two adjacent fractures. Because of the finite fracture length, the late time behaviors of the two models differ. The comparisons of the pressure derivative values from both models are shown in Fig. 12. Our model derivative matches well with the derivative from Kuchuk and Habashy (1997) during short and moderate times, and differs at late times because, as explained above, we used finite-length fractures. The same figure also shows the derivative of the homogenous (background medium) system without fractures. All three curves behave in the same way at early times, but the curves for fractured reservoirs deviate from the homogenous model behavior at $t_{\mathrm{D}} \approx 10^{-4}$. As shown in Fig. 12, the curves for both cases do not show a well-stabilized radial flow regime $(m=0)$ because the 20th fracture, which is $5 \mathrm{ft}$ away from the well, affects the flow behavior of the system significantly. Both derivatives exhibit a flow regime, which looks like a radial flow regime $(m=0)$, from $t_{\mathrm{D}}=0.01$ to $t_{\mathrm{D}}=0.5$, after which the Kuchuk and Habashy (1997) and our model derivatives exhibit $m=1 / 5$ and $m=1 / 3$ slope flow regimes, respectively. As observed in Fig. 12, both models go towards a radial flow regime $(m=0)$ as a result of the background homogenous medium at late times. As noted in the previous paragraph, the incompressible 


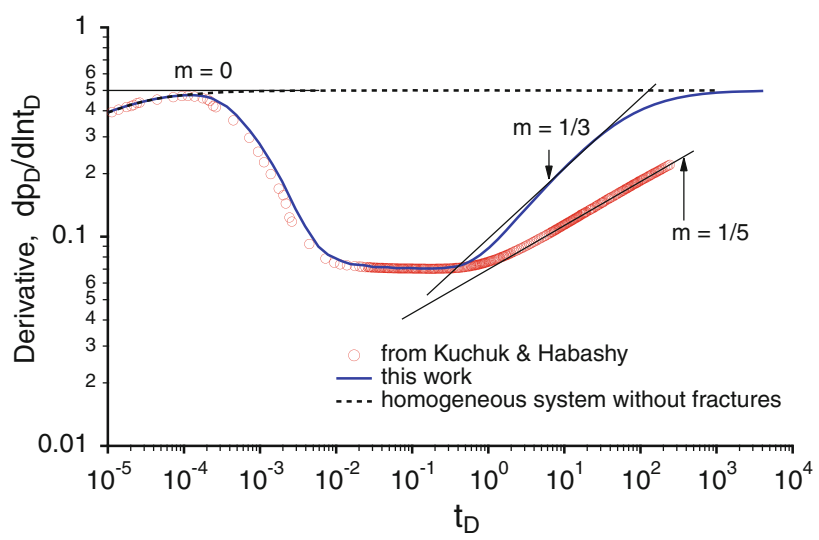

Fig. 12 Comparison of pressure logarithmic derivatives obtained from the Kuchuk and Habashy (1997) solution and the solution given in this article for the model shown in Fig. 11

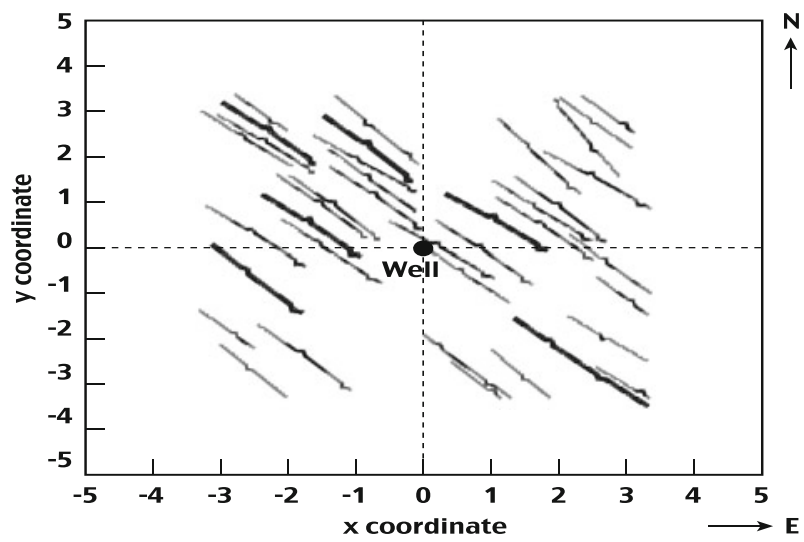

Fig. 13 Distributions of faults or fractures in a discretely fractured reservoir, where the well is located at $(0,0)$

fracture fluid-flow assumption is valid because the Kuchuk and Habashy (1997) compressible flow fracture solution matches well with the incompressible flow fracture solution given in this article.

Finally, we have simulated the transient pressure behavior of a reservoir configuration containing 40 fractures or faults (Fig. 13). In this system, the finite-length geological features in Fig. 13 represent either fractures or faults predominately in the north-east direction, where the background porous medium is homogeneous. Here, we used $r_{\mathrm{Dw}}=0.66 \times 10^{-3}$ and the reference length $l=150 \mathrm{~m}(492.1 \mathrm{ft})$. All faults or fractures are fully penetrating the formation, and are vertical and disjointed. Here, the effects of wellbore storage and skin are intensionally disregarded to observe the full effects of fractures and faults on the behavior of the system.

For the first case, it is assumed that all geological features as shown in Fig. 13 are 40 fractures with the same conductivity. For each run the dimensionless fracture conductivity 


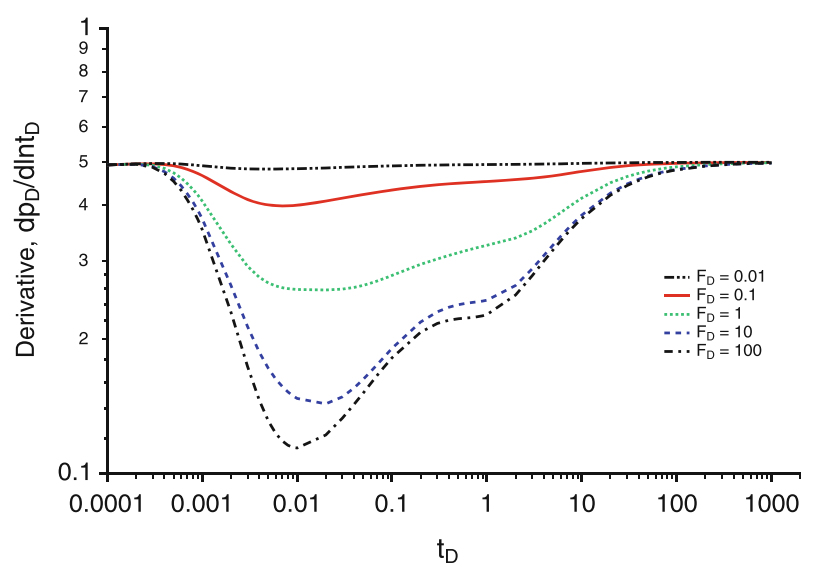

Fig. 14 Dimensionless logarithmic derivative of wellbore pressure for a discretely fractured reservoir shown in Fig. 13

( $F_{\mathrm{D}}$ defined by Eq. 38 ) remains the same, but is varied from $10^{-2}$ to 100 to compute the logarithmic derivatives of the wellbore pressures as shown in Fig. 14. Notice from Fig. 14 that as the value of $F_{\mathrm{D}}$ becomes small, the effects of fractures on the derivative behavior of the system become negligible as can be seen from the $F_{\mathrm{D}}=10^{-2}$ curve. On the other hand, the effects of high conductivity fractures are significant. All these derivative curves for different values of $F_{\mathrm{D}}$, particularly highly conductive ones, weakly resemble the Warren and Root (1963) dual-porosity reservoir model derivative behavior; however, the minimums (dip) and the shape of the derivates at the large times are quite different. Notice also that all derivatives approach 0.5 ( $m=0$ line-radial flow) almost at the same time in spite of the big difference in the fracture conductivities.

For the second case, it is assumed that all geological features as shown in Fig. 13 are 40 faults with the same transmissibility. Again, for each run the dimensionless fault transmissibility ( $\alpha_{D}$ defined by Eq. 59) remains the same but is varied from 0 to 1,000 to compute wellbore pressure logarithmic derivatives for different transmissibility values as shown in Fig. 15. The $\alpha_{\mathrm{D}}=0$ case corresponds to zero fault permeability, $k_{\mathrm{f}}=0$, a sealing fault. The $\alpha_{\mathrm{D}}=0$ curve $\left(\frac{\mathrm{d} p_{\mathrm{D}}}{\mathrm{d} \ln \left(t_{\mathrm{D}}\right)}\right)$ goes above 1 . The derivative value of 1 is the double slope line if the fault is sealing and extends to infinity. If there is only one finite-length sealing fault in the porous media, the derivative should become 1 (broken line in Fig. 14), then the derivative goes to 0.5 ( $m=0$ line-radial flow), which is the derivative of the homogenous background formation. When $\alpha_{\mathrm{D}}=1,000$, the fault permeability becomes almost equal to the formation permeability, then the derivative becomes almost equal to 0.5 ( $m=0$ line) as can be seen in Fig. 15. Notice that the derivative curves for the small values of $\alpha_{\mathrm{D}}$ have a slope of $m=1 / 3$ at early times and have two humps. For the large values of $\alpha_{\mathrm{D}}$, the second hump and the slope of $m=1 / 3$ disappear. All derivatives approach 0.5 (radial flow) almost at the same time in spite of the big difference in the fault transmissibilities.

In some reservoirs, the fault permeabilities could be higher than the host formation permeabilities as discussed above or fracture permeabilities could be lower. Nonetheless, our solutions apply to both low and high conductivity fractures and both low and high transmissibility faults. 


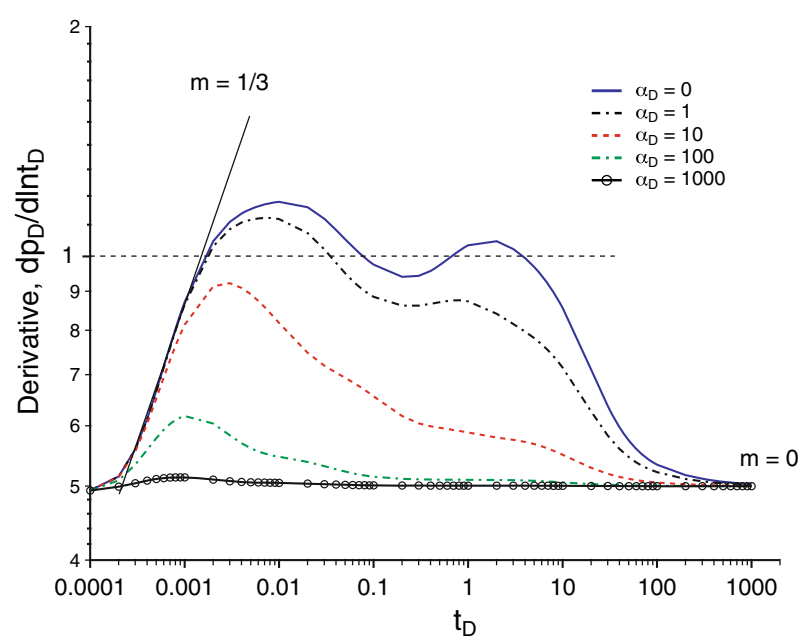

Fig. 15 Dimensionless logarithmic derivative of wellbore pressure for a discretely faulted reservoir shown in Fig. 13

\section{Conclusion}

In this article, we have presented transient pressure solutions for a vertical well in a reservoir containing arbitrary distributed finite- and/or infinite-conductivity faults and/or fractures, i.e., in discretely fractured and faulted reservoirs. These solutions are mesh-free semianalytical in a 2D infinite reservoir and are given both in time and Laplace domains. The particularity of the solutions is that they do not rely on space or time discretization, but rather treat the domain of interest as a whole. This flexibility enables us to obtain the pressure in any point of the spatial domain at any time, and also avoids error accumulation as time increases. It is essentially based on the idea of introduction of unknown flux density functions on fractures/faults or wellbore and their expansion in terms of singular basis functions. It allows to reduce the problem to a system of linear algebraic equations of modest size, which is easy to solve numerically. The solution techniques are numerically stable and computationally efficient. The solutions are extended to account for other reservoir features, such as sealing or leaky faults. Solutions are also presented for the compressible fluid flow inside fractures. It is shown that the compressibility effects are negligible, and the incompressible fluid flow assumption in fractures is correct.

Derivative examples of transient pressure behavior of different discretely fractured and faulted reservoirs are presented. These derivatives can be used as diagnostic tools for well test interpretation. It is shown that discretely fractured reservoirs do not exhibit well-known characteristics of the Warren and Root (1963) dual-porosity reservoir model.

Acknowledgments The authors are grateful to Schlumberger for permission to publish this article, and would like to thank Dr. Jacob Bear, Technion-Israel Institute of Technology, and Dr. Atilla Aydin, Stanford University, California, for valuable discussions of fractured media. We also thank Amine Ennaifer, Kirsty Morton, and Richard Booth, Schlumberger, for valuable discussions of naturally fractured reservoirs.

Open Access This article is distributed under the terms of the Creative Commons Attribution License which permits any use, distribution, and reproduction in any medium, provided the original author(s) and the source are credited. 


\section{Appendix}

Here, we explain how to compute the expansion functions defined by Eq. 31 efficiently. Except on the generating fracture itself these functions can be easily evaluated using Chebyshev-Gauss quadrature which gives very high precision even for a small number of nodes:

$$
\begin{aligned}
\bar{p}_{\mathrm{D} i n}(x, y, s) & =\frac{1}{\pi} \int_{0}^{\pi} \mathrm{K}_{0}\left[\sqrt{s} l_{\mathrm{D} i} \sqrt{\left(x_{\mathrm{D} i}-\cos \psi\right)^{2}+y_{\mathrm{D} i}^{2}}\right] \cos n \psi \mathrm{d} \psi \\
& \approx \frac{1}{N} \sum_{k=0}^{N} \mathrm{~K}_{0}\left[\sqrt{s} l_{\mathrm{D} i} \sqrt{\left(x_{\mathrm{D} i}-\cos \psi_{k}\right)^{2}+y_{\mathrm{D} i}^{2}}\right] \cos n \psi_{k},
\end{aligned}
$$

where $\psi_{k}=\frac{\pi}{N}(0.5+k), k=0,1, \ldots, N-1$. Notice that $N$ should be greater than $n$, although, since the higher $n$ functions decay very fast as distance from fracture increases, we can take lesser $N$ and just neglect $\bar{p}_{\text {Din }}$ with $n>N$ assuming them to be zero. It seems that the optimal value of $N$ in terms of precision/speed is 60 for the points close to the generating fracture $\left(x_{\mathrm{D} i}^{2}+y_{\mathrm{D} i}^{2}<1\right)$ and then it can be reduced to 30 and even further down to $N=1$ for $\sqrt{x_{\mathrm{D} i}^{2}+y_{\mathrm{D} i}^{2}}>10$, without almost any loss in accuracy. Since on the fracture itself the sub-integral function becomes singular the direct use of this quadrature rule is inefficient, but the singularity can be easily removed if we rewrite $\bar{p}_{\text {Din }}$ as

$$
\begin{aligned}
& \bar{p}_{\mathrm{Din}}(\cos \chi, 0, s) \\
& =\frac{1}{\pi} \int_{0}^{\pi}\left[\mathrm{K}_{0}\left(\sqrt{s} l_{\mathrm{D} i}|\cos \chi-\cos \psi|\right)+\ln \left(\frac{\sqrt{s} l_{\mathrm{D} i}}{2}|\cos \chi-\cos \psi|\right)+\gamma\right] \cos n \psi \mathrm{d} \psi \\
& \quad-\left[\ln \left(\frac{\sqrt{s l_{\mathrm{D} i}}}{2}\right)+\gamma\right] \delta_{0 n}-\frac{f_{n}(\chi)}{\pi},
\end{aligned}
$$

where

$$
f_{n}(\chi)=\int_{0}^{\pi} \ln |\cos \chi-\cos \psi| \cos n \psi \mathrm{d} \psi=\left\{\begin{array}{l}
-\pi \ln 2, \quad \text { if } n=0, \\
-\frac{\pi}{n} \cos n \chi, \quad \text { if } n>0 .
\end{array}\right.
$$

Now sub-integral function becomes non-singular, so it can be evaluated easily using Chebyshev-Gauss quadrature rule. The same approach can be applied for numerical evaluation of integrals in Eqs. 61, 65 which define the expansion functions for faults. In this case, the pressure change on the fault is expanded in terms of Gegenbauer polynomials, so it might be more efficient to use Gegenbauer-Gauss quadrature rule which is described, for example, in Stroud and Secrest (1966). One can also evaluate the integrals in Eqs. 70 and 73 in the same way. Since we did not specify any particular expansion functions for boundaries, it is difficult to suggest any specific quadrature rule, but if one decides to use trigonometric functions as basis on the boundary, the Chebyshev-Gauss quadrature rule seems to provide very good results in terms of speed and precision.

The same techniques can be also applied to optimize the computations of similar integrals containing exponential integral functions $\mathrm{E}_{1}$ and $\mathrm{E}_{2}$ in time domain. 


\section{References}

Abbaszadeh, M., Asakawa, K., Cinco-Ley, H., Arihara, N.: Interference testing in reservoirs with conductive faults or fractures. SPE Reserv. Eval. Eng. 3(5), 426-434 (2000)

Abramowitz, M., Stegun, I.A.: Handbook of Mathematical Functions. Dover, New York (1972)

Aydin, A.: Fractures, faults, and hydrocarbon migration and flow. Mar. Pet. Geol. 17, 797-814 (2000)

Barenblatt, G.I., Zeltov, Y.P., Kochina, I.: Basic concepts in the theory of seepage of homogeneous liquids in fissured rocks. PMM (J. Sov. Appl. Math. Mech.) 24(5), 1286-1303 (1960)

Bogdanov, I.I., Mourzenko, V.V., Thovert, J.-F., Adler, P.M.: Pressure drawdown well tests in fractured porous media. Water Resour. Res., 39(1), 1021 (2003)

Brebbia, C.A.: The Boundary Element Method for Engineers. Halstead Press, New York (1978)

Cinco-Ley, H., Samaniego, F.V.: Transient pressure analysis for fractured wells. J. Pet. Technol. 33(9), 1749-1766 (1981)

Cinco-Ley, H., Samaniego, F.V., Dominguez, N.: Unsteady state behavior for a well near a natural fracture. SPE 6019, SPE Annual Technical Conference and Exhibition, New Orleans (1976)

Cinco-Ley, H., Samaniego, F.V., Dominguez, N.A.: Transient pressure behavior for a well with a finite-conductivity vertical fracture. Soc. Pet. Eng. J. 18, 253-264 (1978)

Flodin, E., Aydin, A., Durlofsky, L., Yeten, B.: Representation of fault zone permeability in reservoir flow models. Paper SPE 71617, SPE Annual Technical Conference and Exhibition, 30 Sept-3 Oct, New Orleans, Louisiana (2001)

Horner, D.: Pressure buildup in wells. In: Brill, E.J. (ed.) Proceedings, Leiden. Third World Petroleum Congress (1951)

Jourde, H., Flodin, E.A., Aydin, A., Durlofsky, L.J., Wen, X.-H.: Computing permeability of fault zones in eolian sandstone from outcrop measurements. AAPG Bull. 86(7), 1187-1200 (2002)

Kuchuk, F., Habashy, T.: Pressure behavior of laterally composite reservoirs. SPE Form. Eval. 12(1), 47-56 (1997)

Lee, S.-T., Brockenbrough, J.R.: A new approximate analytic solution for finite-conductivity vertical fractures. SPE Form. Eval. 1(1), 75-88 (1986)

Matthai, S.K., Aydin, A., Pollard, D.D., Roberts, S.: Simulation of transient well-test signatures for geologically realistic faults in sandstone reservoirs. SPE J. 3(1), 62-76 (1998)

Nelson, R.A.: Geologic Analysis of Naturally Fractured Reservoirs. Gulf Publishing, Houston (1985)

Prasad, R.: Pressure transient analysis in the presence of two intersecting boundaries. J. Pet. Technol. 27(1), 89-96 (1975)

Prudnikov, A., Brychkov, Y., Marichev, O.I.: Integrali i ryadi. Elementarnie funkcii (in Russian). Nauka, Moscow (1981)

Riley, M., Brigham, W., Horne, R.: Analytic solutions for elliptical finite-conductivity fractures. Paper SPE 22656, SPE Annual Technical Conference and Exhibition, 6-9 October 1991, Dallas, Texas (2007)

Ruijtenberg, P., Buchanan, R., Marke, P.: Three-dimensional data improve reservoir mapping. J. Pet. Technol. 42(1), 22-25 (1990)

Samaniego, F.V., Cinco-Ley, H.: Transient well testing, chap. 10—naturally fractured reservoirs. M. Kamal (ed.) Monograph Series 23, 1st edn, pp. 221-280. Society of Petroleum Engineers, Dallas (2009)

Stroud, A.H., Secrest, D.: Gaussian Quadrature Formulas. Prentice-Hall, Englewood Cliffs (1966)

Tiab, D., Kumar, A.: Detection and location of two parallel sealing faults around a well. J. Pet. Technol. 32(10), 1701-1708 (1980)

Warren, J.E., Root, P.J.: The behavior of naturally fractured reservoirs. SPE J. 3(3), 245-255 (1963)

Yaxley, L.M.: Effect of a partially communicating fault on transient pressure behaviour. SPE Form. Eval. 2(6), 590-598 (1978) 